\title{
THE CONTRIBUTION OF HALOS WITH DIFFERENT MASS RATIOS TO THE OVERALL GROWTH OF CLUSTER-SIZED HALOS
}

\author{
Doron Lemze ${ }^{1}$, Marc Postman ${ }^{2}$, Shy Genel $^{3}$, Holland C. Ford ${ }^{1}$, Italo Balestra $^{4,5}$, Megan Donahue ${ }^{6}$, Daniel Kelson ${ }^{7}$, \\ Mario Nonino ${ }^{4}$, Amata Mercurio ${ }^{5}$, Andrea Biviano ${ }^{4}$, Piero Rosati ${ }^{8}$, Keitchi Umetsu ${ }^{9}$, David Sand $^{10}$, \\ Anton Koekemoer ${ }^{2}$, Massimo Meneghetti ${ }^{11,12}$, Peter Melchior $^{13,14}$, Andrew B. Newman $^{15}$, Waqas A. Bhatti $^{16}$, \\ G. Mark Voit ${ }^{7}$, Elinor Medezinski ${ }^{1}$, Adi Zitrin ${ }^{17}$, Wei Zheng ${ }^{1}$, Tom Broadhurst ${ }^{18,19}$, Matthias Bartelmann ${ }^{17}$, \\ Narciso Benitez ${ }^{20}$, Rychard Bouwens ${ }^{21}$, Larry Bradley ${ }^{2}$, Dan CoE ${ }^{2}$, Genevieve Graves ${ }^{16,22}$, Claudio Grillo ${ }^{23}$, \\ Leopoldo Infante $^{24}$, Yolanda Jimenez-Teja ${ }^{20}$, Stephanie Jouvel ${ }^{25}$, Ofer Lahav ${ }^{26}$, Dan MaOz ${ }^{27}$, Julian Merten ${ }^{28}$, \\ Alberto Molino $^{20}$, John Moustakas $^{29}$, LeOnidas Moustakas $^{28}$, Sara OGaz $^{2}$, Marco ScodegGio $^{30}$, and Stella Seitz ${ }^{31,32}$ \\ ${ }^{1}$ Department of Physics \& Astronomy, Johns Hopkins University, 3400 North Charles Street, Baltimore, MD 21218, USA \\ 2 Space Telescope Science Institute, 3700 San Martin Drive, Baltimore, MD 21208, USA \\ ${ }^{3}$ Harvard-Smithsonian Center for Astrophysics, 60 Garden Street, Cambridge, MA 02138, USA \\ ${ }^{4}$ INAF/Osservatorio Astronomico di Trieste, via G.B. Tiepolo 11, I-34143 Trieste, Italy \\ ${ }^{5}$ INAF/Osservatorio Astronomico di Capodimonte, Salita Moiariello 16, I-80131 Napoli, Italy \\ ${ }^{6}$ Department of Physics and Astronomy, Michigan State University, East Lansing, MI 48824-2320, USA \\ ${ }^{7}$ Carnegie Institute for Science, Carnegie Observatories, Pasadena, CA, USA \\ ${ }^{8}$ European Southern Observatory, Karl-Schwarzschild Strasse 2, D-85748 Garching, Germany \\ ${ }^{9}$ Institute of Astronomy and Astrophysics, Academia Sinica, P.O. Box 23-141, Taipei 10617, Taiwan \\ ${ }^{10}$ Department of Physics, Texas Tech University, Box 41051, Lubbock, TX 79409-1051, USA \\ ${ }^{11}$ INAF, Osservatorio Astronomico di Bologna, via Ranzani 1, I-40127 Bologna, Italy \\ 12 INFN, Sezione di Bologna, viale Berti Pichat 6/2, I-40127, Bologna, Italy \\ ${ }^{13}$ Center for Cosmology and Astro-Particle Physics, The Ohio State University, 191 West Woodruff Avenue, Columbus, OH 43210, USA \\ ${ }^{14}$ Department of Physics, The Ohio State University, 191 West Woodruff Avenue, Columbus, OH 43210, USA \\ ${ }^{15}$ Cahill Center for Astronomy and Astrophysics, California Institute of Technology, MS 249-17, Pasadena, CA 91125, USA \\ ${ }^{16}$ Department of Astrophysical Sciences, Princeton University, Princeton, NJ 08544, USA \\ ${ }^{17}$ Institut für Theoretische Astrophysik, Zentrum für Astronomie, Universität Heidelberg, Philosophenweg 12, D-69120 Heidelberg, Germany \\ ${ }^{18}$ Department of Theoretical Physics, University of Basque Country UPV/EHU, E-48080 Bilbao, Spain \\ ${ }^{19}$ IKERBASQUE, Basque Foundation for Science, E-48011 Bilbao, Spain \\ ${ }^{20}$ Instituto de Astrofisica de Andalucia (CSIC), Glorieta de la Astronomia s/n, E-18008 Granada, Spain \\ ${ }^{21}$ Leiden Observatory, Leiden University, NL-2333 Leiden, The Netherlands \\ ${ }^{22}$ Department of Astronomy, University of California, Berkeley, CA, USA \\ ${ }^{23}$ Dark Cosmology Centre, Niels Bohr Institute, University of Copenhagen, DK-2100 Copenhagen, Denmark \\ 24 Institute of Astrophysics and Center for Astroengineering, Pontificia Universidad Católica de Chile, Santiago, Chile \\ ${ }^{25}$ Institut de Cincies de l'Espai (IEE-CSIC), E-08193 Bellaterra (Barcelona), Spain \\ ${ }^{26}$ Department of Physics and Astronomy, University College London, London, UK \\ 27 The School of Physics and Astronomy, Tel Aviv University, Tel Aviv, Israel \\ 28 Jet Propulsion Laboratory, California Institute of Technology, Pasadena, CA 91109, USA \\ ${ }^{29}$ Department of Physics and Astronomy, Siena College, Loudonville, NY, USA \\ ${ }^{30}$ INAF-IASF Milano, Via Bassini 15, I-20133 Milano, Italy \\ ${ }^{31}$ Instituts für Astronomie und Astrophysik, Universitäs-Sternwarte München, D-81679 München, Germany \\ 32 Max-Planck-Institut für extraterrestrische Physik (MPE), D-85748 Garching, Germany \\ Received 2013 March 20; accepted 2013 August 3; published 2013 October 3
}

\begin{abstract}
We provide a new observational test for a key prediction of the $\Lambda$ CDM cosmological model: the contributions of mergers with different halo-to-main-cluster mass ratios to cluster-sized halo growth. We perform this test by dynamically analyzing 7 galaxy clusters, spanning the redshift range $0.13<z_{\mathrm{c}}<0.45$ and caustic mass range 0.4-1.5 $10^{15} h_{0.73}^{-1} M_{\odot}$, with an average of 293 spectroscopically confirmed bound galaxies to each cluster. The large radial coverage (a few virial radii), which covers the whole infall region, with a high number of spectroscopically identified galaxies enables this new study. For each cluster, we identify bound galaxies. Out of these galaxies, we identify infalling and accreted halos and estimate their masses and their dynamical states. Using the estimated masses, we derive the contribution of different mass ratios to cluster-sized halo growth. For mass ratios between $\sim 0.2$ and $\sim 0.7$, we find a $\sim 1 \sigma$ agreement with $\Lambda$ CDM expectations based on the Millennium simulations I and II. At low mass ratios, $\lesssim 0.2$, our derived contribution is underestimated since the detection efficiency decreases at low masses, $\sim 2 \times 10^{14} h_{0.73}^{-1} M_{\odot}$. At large mass ratios, $\gtrsim 0.7$, we do not detect halos probably because our sample, which was chosen to be quite X-ray relaxed, is biased against large mass ratios. Therefore, at large mass ratios, the derived contribution is also underestimated.
\end{abstract}

Key words: dark matter - galaxies: clusters: individual (Abell 611, Abell 963, Abell 1423, Abell 2261, MACS J1206.2-0848, RX J2129.7+0005, CL 2130.4-0000) - galaxies: kinematics and dynamics

Online-only material: color figures, machine-readable tables 


\section{INTRODUCTION}

$\Lambda \mathrm{CDM}$ makes clear predictions for the growth of mass in halos due to accretion of other halos with different masses (Fakhouri \& Ma 2008, hereafter FM08; Berrier et al. 2009; Fakhouri et al. 2010, hereafter FMB10; Genel et al. 2010, hereafter G10). For example, G10, who used several $N$-body simulations to construct merger trees while taking special care of halo fragmentation, suggested that mergers with mass ratios larger than $1 / 3(1 / 10)$ contribute $\approx 20 \%(\approx 30 \%)$ of the total halo mass growth.

Confirming these predictions observationally is an important test for the cosmological model. Using spectroscopically identified galaxies that cover the whole cluster infall region is ideal for this purpose. The three-dimensional (3D) spectroscopic data (2D spatial information plus the redshift data) enable identification of bound galaxies (den Hartog \& Katgert 1996, hereafter HK96; Diaferio 1999, hereafter D99). Then, out of all the bound galaxies, one can identify the cluster and the infalling and accreted satellite halos. The mass ratios between the cluster and these identified satellite halos can reach low values, e.g., $\sim 1 / 100$ (Adami et al. 2005; this work), which enable a wide range for comparing the estimated growth of cluster mass due to mergers with different mass ratios and the theoretical predicted one.

In a previous work, Adami et al. (2005) identified 17 groups within the Coma cluster. They grossly estimated these groups' masses and survival times in the cluster. Then they estimated that at least $\sim 10 \%-30 \%$ of the cluster mass was accreted since $z \sim 0.2-0.3$ by halos with mass ratios larger than about $1 / 100$, in agreement with the theoretical prediction. However, since the simulation-based predictions are made by averaging over a large number of clusters, we need to compare these predictions with estimations made from a cluster sample. We also need to observe the halos while they infall and accrete in order to follow the cluster mass accretion process itself.

In this work, we first identify and exclude unbound galaxies from a sample of seven galaxy clusters. We measure the clusters' masses using different mass estimators and derive various mass uncertainties. Then, out of all the bound galaxies, we also define infalling and accreted ${ }^{33}$ satellite halos and estimate their masses and dynamical states. We use the measured masses of both the satellites and clusters to estimate the growth of cluster-sized halos via the accretion of smaller halos. Finally, we compare our observational constraints of cluster growth to those from simulations.

The Cluster Lensing and Supernova survey with Hubble (CLASH; Postman et al. 2012, hereafter P12) is a large Hubble program imaging 25 galaxy clusters. This cluster survey is also covered by supporting observations from a large number of space- and ground-based telescopes, enabling an unprecedented multi-wavelength study of clusters. Extensive groundbased spectroscopy for galaxies in the environs of the CLASH clusters either was available from the Sloan Digital Sky Survey (SDSS; Stoughton et al. 2002) and the Hectospec Cluster Survey (HeCS; Rines et al. 2013) or was initiated specifically in support of the CLASH program using the VLT/VIMOS and Magellan/IMACS instruments. The large radial coverage (a few virial radii), which covers the whole infall region, with a high number of spectroscopically identified galaxies

\footnotetext{
33 In some cases, the identified accreted satellite halos are fully within the cluster virial radius and can be considered as substructure. However, in this work we consider them as accreted satellites.
}

enables this new study. In this paper, we study the dynamics of a subset of the CLASH clusters: Abell 611 (hereafter A611), Abell 1423 (hereafter A1423), Abell 2261 (hereafter A2261), MACS J1206.2-0848 (hereafter MACSJ1206), and RX J2129.7+0005 (hereafter RXJ2129). In addition, we also analyze CL 2130.4-0000 (hereafter CL2130), which is in the foreground of RXJ2129, and Abell 963 (hereafter A963), which was originally in the CLASH sample but was replaced by A1423 when it was found that A963 could not be scheduled for Hubble Space Telescope (HST) observations due to a lack of usable guide stars.

The paper is organized as follows. In Section 2, we present a short description of the data we used and their reduction process. In Section 3, we describe the methods and tests used to identify non-cluster members using the spectroscopic data, estimate halos' masses, identify halos, and estimate halos' substructure levels and relaxation states. In Section 4, we calculate the simulation-based expected fraction of cluster mass accretion. In Section 5, we show our results: in Section 5.1 we present the derived mass profiles, in Section 5.2 we estimate the clusters' dynamical state, in Section 5.3 we show our findings regarding the growth of cluster-sized halos, and in Section 5.4 we show our estimations for identified satellites' substructure level and relaxation state and the correlation between them. We discuss our results in Section 6 and summarize them in Section 7.

Unless explicitly mentioned, the cosmology used throughout this paper is WMAP7 (Komatsu et al. 2011), i.e., $\Omega_{\mathrm{m}}=0.272$, $\Omega_{\Lambda}=0.728$, and $h \equiv 0.73 h_{0.73}=0.73$ where $H_{0}=$ $100 \mathrm{~h} \mathrm{~km} \mathrm{~s}^{-1} \mathrm{Mpc}^{-1}$. Errors represent a confidence level of $68.3 \%(1 \sigma)$.

\section{CLUSTER SAMPLE AND OBSERVATIONAL DATA}

The sample selected for the dynamical analyses here consists of seven clusters with extensive spectroscopic redshift measurements. These clusters are the first ones we acquired (out of about the $20 \mathrm{X}$-ray selected) in the CLASH project with a high number (a few hundred) of cluster members and infalling galaxies. The clusters' locations and redshifts are given in Section 5 (where we derive most of them). For more of their X-ray properties (except for CL2130), see P12 (Table 4).

\subsection{Spectroscopy}

Spectra for galaxies in the environs of each cluster were drawn from a combination of existing data and new observations. For A611, A963, A1423, A2261, RXJ2129, and CL2130 the bulk of the redshifts were obtained using the Hectospec instrument on the MMT (Fabricant et al. 2005). The sample selection, observational parameters, and data reduction procedures for the Hectospec data are described in detail in Rines et al. (2013), except for $\sim 50 \%$ of A611 redshifts, which were first used in Newman et al. (2013a, 2013b). Hectospec's circular $1^{\circ}$ diameter field of view covers the entire virial region and a significant fraction of the infall region of the above clusters in a single pointing. The SDSS DR7 release (Abazajian et al. 2009) was used to provide additional redshift information for 439 galaxies.

In Tables 1 and 2, we present the MMT/Hectospec redshifts for CL2130 and A611, respectively. In these tables, Columns 1 and 2 list the galaxy's equatorial coordinates (in degrees) for epoch J2000.0. Columns 3 and 4 list the heliocentric redshift and the redshift error, respectively. The fifth and sixth columns contain the Tonry cross-correlation coefficient (Tonry \& Davis $1979), R_{\text {cross }}$, and redshift quality flag, respectively. The quality 
Table 1

CL2130 MMT/Hectospec Spectroscopic Redshifts

\begin{tabular}{lcccr}
\hline \hline $\begin{array}{l}\text { R.A. } \\
(\mathrm{J} 2000 \mathrm{deg})\end{array}$ & $\begin{array}{c}\text { Decl. } \\
(\mathrm{J} 2000 \mathrm{deg})\end{array}$ & $z$ & $\Delta z$ & $R_{\text {cross }}$ \\
\hline 322.6146364 & -0.0816536 & 0.134668 & 0.000058 & 13.64 \\
322.7121945 & -0.2050228 & 0.135396 & 0.000156 & 7.07 \\
322.3012405 & -0.3572667 & 0.136382 & 0.000153 & 7.33 \\
322.2246194 & -0.0703775 & 0.184074 & 0.000120 & 8.04 \\
322.3168903 & -0.0935747 & 0.143829 & 0.000054 & 21.69 \\
\hline
\end{tabular}

(This table is available in its entirety in a machine-readable form in the online journal. A portion is shown here for guidance regarding its form and content.)

flags were assigned by Rines et al. (2013) to each spectral fit. The flags are "Q" for high-quality redshifts, "?" for marginal cases, and " $\mathrm{X}$ " for poor fits. Although repeated observations of several targets with "?" flags show that these redshifts are generally reliable, we use only the high-quality redshifts in this paper. The MMT/Hectospec redshifts for A963, A1423, A2261, and RXJ2129 are published in Rines et al. (2013).

The vast majority (2485) of the 2535 galaxy redshifts we use for MACSJ1206 were obtained using the VIMOS instrument (Le Fèvre et al. 2003) on the VLT in multi-object spectroscopy mode. We consider only the VLT highly reliable, $\gtrsim 80 \%$, redshift estimates. The VIMOS data were acquired using four separate pointings, always keeping one quadrant centered on the cluster core. This strategy allows us to get spectra for fainter arcs in the core down to $R \approx 25.5 \mathrm{mag}$ and to a $\sim 80 \%$ success limit of $R \approx 24.5$ in the non-overlapping regions. A broad spectral range, from $370 \mathrm{~nm}$ to $970 \mathrm{~nm}$, was achieved by using the LR blue and the MR grisms, which yield spectral resolutions of 180 and 580, respectively. For more details about the VIMOS target selection, survey design, and data, see P. Rosati et al. (in preparation).

We specifically targeted galaxies within the core of the MACSJ1206 using the Inamori Magellan Areal Camera and Spectrograph (Dressler et al. 2011) on the Baade $6.5 \mathrm{~m}$ telescope. We utilized the Gladders Image-Slicing Multislit Option, which reformats a $4^{\prime} \times 4^{\prime}$ field over a wider area of the telescope focal plane, thus enabling a large increase in the multiplexing capability of the instrument. We accumulated 210 minutes of exposure time in six exposures with the $300 \mathrm{~mm}^{-1}$ grism, with a dispersion of $\sim 1.34 \AA$ and a resolution of $\sim 5 \AA$ (FWHM). We obtained redshifts for 21 galaxies to $I_{\mathrm{F} 814 \mathrm{~W}}=22 \mathrm{mag}$.

The remaining 29 redshifts in MACSJ1206 were taken from the literature (Jones et al. 2004, 2009; Lamareille et al. 2006; Ebeling et al. 2009).

For the target selection plane of the sky completeness, see Appendix A.

\subsection{X-Ray Profiles}

We derived cumulative mass profiles from the public ACCEPT (Cavagnolo et al. 2009) intracluster medium projected temperature and deprojected density profiles by assuming that the hot gas is in hydrostatic equilibrium with a spherically symmetric cluster gravitational potential. These temperature and density profiles were derived from archival Chandra data by Cavagnolo et al. (2009). Small calibration differences may change the absolute mass estimates by a small amount, but within the uncertainty. The temperature profile was interpolated to the resolution of the density profiles (as in Cavagnolo et al.). Some regularization of the resulting pressure profiles was re-
Table 2

A611 MMT/Hectospec Spectroscopic Redshifts

\begin{tabular}{lcccc}
\hline \hline $\begin{array}{l}\text { R.A. } \\
(J 2000 \mathrm{deg})\end{array}$ & $\begin{array}{c}\text { Decl. } \\
(\mathrm{J} 2000 \mathrm{deg})\end{array}$ & $z$ & $\Delta z$ & $R_{\text {cross }}$ \\
\hline 120.2032223 & 36.1133006 & 0.144207 & 0.000023 & 19.06 \\
120.0800936 & 36.4262502 & 0.257982 & 0.000108 & 13.33 \\
119.8793292 & 36.3154402 & 0.176505 & 0.000075 & 17.47 \\
120.1680427 & 36.1790189 & 0.503699 & 0.000115 & 11.16 \\
119.7670295 & 36.4042486 & 0.271796 & 0.000080 & 19.48 \\
\hline
\end{tabular}

(This table is available in its entirety in a machine-readable form in the online journal. A portion is shown here for guidance regarding its form and content.)

quired for stable estimates of errors on the pressure gradients, so we applied a minimal requirement that the shape of the pressure profile followed the "universal" pressure profile derived by Arnaud et al. (2010). We did not assume an NFW (Navarro et al. 1997 ) profile or a density radial profile (such as a beta law). Best estimates and $1 \sigma$ uncertainties for the cumulative mass within each radius were estimated using a simulated annealing technique (Kirkpatrick et al. 1983). These profiles are consistent with mass profiles derived using other techniques (e.g., Umetsu et al. 2012; Donahue et al., in preparation).

\section{METHODS AND TESTS}

We describe the methods we use to identify galaxies that are bound to the cluster, define (from the bound galaxies) satellites' in the plane of the sky, derive the cluster and infalling and accreted satellites' mass estimates and the corresponding uncertainties in those estimates, and estimate the level of substructure and relaxation state of each halo.

\subsection{Determining the Cluster Redshift}

We begin by obtaining an estimate for the mean redshift of each cluster, $z_{\mathrm{c}}$. To estimate $z_{\mathrm{c}}$, we first select galaxies that lie within a projected radius of $10 h_{0.73}^{-1} \mathrm{Mpc}$ from the cluster center, which is chosen to be the peak of the cluster's X-ray surface brightness distribution. We adopt an initial guess for $z_{\mathrm{c}}$ by fitting a Gaussian to the largest peak in the redshift histogram of the galaxies lying within the above projected radius. Velocity offsets from the mean cluster redshift are defined for each galaxy as $v=c\left(z-z_{\mathrm{c}}\right) /\left(1+z_{\mathrm{c}}\right)$ (Harrison \& Noonan 1979).

We then make an initial cut based on this velocity offset, excluding all galaxies with $|v|>4000 \mathrm{~km} \mathrm{~s}^{-1}$. A histogram of the redshifts of the galaxies with $|v| \leqslant 4000 \mathrm{~km} \mathrm{~s}^{-1}$ is then generated, using a velocity bin size of $150 \mathrm{~km} \mathrm{~s}^{-1}$ (except for MACSJ1206, where it is $200 \mathrm{~km} \mathrm{~s}^{-1}$ ). This bin size is larger than any of the redshift measurement uncertainties used in this work (except for a few in MACSJ1206 which are not taken into consideration here). The galaxy number uncertainty in each bin was derived assuming Poisson statistics, i.e., $\Delta N=\sqrt{N}$. Poisson uncertainty of zero counts was defined as 1 . We then refine our estimate of the mean cluster redshift by fitting a Gaussian, $G_{1}$, to the redshift histogram. We then see how the goodness of fit changes by adding in additional Gaussians to the fit:

$$
N(v)=G_{1}(v)+\sum_{i=2}^{N} G_{i}(v),
$$

where $G_{i}=A_{i} \exp \left(-\left(v-\bar{v}_{i}\right)^{2} / 2 \sigma_{i}^{2}\right)$, where $A_{i}, \bar{v}_{i}$, and $\sigma_{i}$ are the Gaussian $i$ normalization, average, and dispersion, 
respectively, and they all are taken to be free parameters. The main cluster peak corresponds to $i=1$. If an addition of a subsequent Gaussian decreases the $\chi_{r}^{2}$, it is added.

The cluster's redshift is then determined to be the average value of the cluster's halo Gaussian. Later in the paper (see Table 3), this value is compared with the median redshift of the galaxies found using D99's interloper removal method (see Appendix B.2). It is important to note that the results of the above Gaussian/Gaussians fitting are only used for determining $z_{\mathrm{c}}$ and not for any other dynamical or mass estimation application.

\subsection{Removal of Non-cluster Galaxies}

Due to projection, any cluster dynamical data sample inevitably contains galaxies that are not bound to the cluster, i.e., interlopers. Removing them is important for accurately estimating the dynamical cluster properties. For removing them, a velocity-space diagram is constructed where the galaxies' lineof-sight (measured with respect to the cluster's mean redshift, hereafter LOS) velocities, $v$, are plotted versus their projected distances from the cluster center, $R$. For a well-defined cluster, the galaxies should be distributed in a characteristic "trumpet" shape, the boundaries of which are termed caustics (Kaiser 1987; Regos \& Geller 1989). Galaxies that are outside the caustics are considered to be interlopers. We test two widely used interloper removal techniques that take into account the combined position and velocity information to estimate caustic location. The first method relies on calculating the maximum LOS velocity that a galaxy may be observed to have (HK96). The second relies on first arranging the galaxies in a binary tree according to a hierarchical method to determine the velocity dispersion and mean projected distance of the members, and then estimating membership by the escape velocity (Diaferio \& Geller 1997; D99). Both methods assume spherical symmetry. For consistency with other works, in the rest of this paper, the caustic notation refers only to the second method. We briefly describe the two methods in Appendix B.

\subsection{Mass Estimators}

In this section, we briefly describe the three different dynamically based mass estimators used in this work. The use of different mass estimators shows the uncertainties due to the mass profile estimator picked and increases the reliability of our results. In Appendix $\mathrm{C}$, we examine various possible mass profile biases, irrespective of the mass estimator used.

\subsubsection{Virial and Projected Mass Estimators}

Two widely used mass estimators are the virial and projected mass estimators. Both of them are derived from the collisionless Boltzmann equation assuming that the system is in steady state and spherical symmetry. Further assumptions are that galaxies trace the dark matter (hereafter DM) distribution and that all galaxies have the same mass (Bahcall \& Tremaine 1981, and references within; Heisler et al. 1985; Binney \& Tremaine 2008).

The virial mass profile estimator is

$$
M_{\mathrm{v}}(\leqslant r) \approx M_{\mathrm{v}}(\leqslant R)=\frac{3 \pi N}{2 G} \frac{\sum_{i}^{N}\left(v_{i}-\bar{v}\right)^{2}}{\sum_{i<j}^{N} 1 / R_{i j}},
$$

where $r$ is the distance to the cluster center, $R$ is the projection of $r, N$ is the number of cluster galaxies inside $R, v$ is the LOS velocity, and $R_{i j}$ are projected distances of galaxy pairs within a cylinder of radius $R$ around the center.
The projected mass profile estimator, ${ }^{34}$

$$
M_{\text {proj }}(\leqslant r) \approx M_{\text {proj }}(\leqslant R)=\frac{f_{\mathrm{PM}}}{\pi G} \sum_{i}^{N} R_{i}\left(v_{i}-\bar{v}\right)^{2} / N,
$$

is more robust in the presence of close pairs because it sums $R$ rather than $1 /(1 / R)$ (Bahcall \& Tremaine 1981). The disadvantage of the projected mass estimator is that it requires defining a center. The constant of proportionality $f_{\mathrm{PM}}$ depends on the distribution of orbits, where $f_{\mathrm{PM}}=64 / \pi$, and $32 / \pi$, and $16 / \pi$ for radial, isotropic, and circular orbits, respectively (Heisler et al. 1985; Rines et al. 2003). Because both radial and circular orbits are considered, we set $f_{\mathrm{PM}}=32$ throughout this paper.

Halos are not isolated systems since matter is continuously falling onto them. This infalling matter was claimed to have a significant overall contribution to the pressure at the halos' boundaries (Shaw et al. 2006; Davis et al. 2011). Therefore, the usual formula of the virial theorem $2 T+U=0$ (Binney \& Tremaine 2008) should be replaced by $2 T+U=3 P V$, where $3 P V$ is the surface pressure term (Chandrasekhar 1961; The \& White 1986; Carlberg et al. 1996, 1997; Shaw et al. 2006; Davis et al. 2011; Lemze et al. 2012). If the pressure term is not taken into consideration, the mass is overestimated. In particular we assume that mass follows the galaxy distribution and follow Girardi et al. (1998), so the corrected virial/projected mass profile, $M_{\mathrm{Cv} / \mathrm{Cproj}}$, is

$$
M_{\mathrm{Cv} / \mathrm{Cproj}}=M_{\mathrm{v} / \text { proj }}\left\{1-4 \pi b^{3} \frac{\rho(b)}{\int_{0}^{b} 4 \pi r^{2} \rho d r}\left[\frac{\sigma_{\mathrm{r}}(b)}{\sigma(<b)}\right]^{2}\right\} \text {, }
$$

where $\rho(r)$ is the cluster mass density, $\sigma(<b)$ refers to the integrated velocity dispersion within the boundary radius $b$, and $\sigma_{\mathrm{r}}(b)$ is the radial velocity dispersion at $b$. Assuming that the velocity anisotropy is constant (as assumed for deriving the projected mass estimator) and in the limiting cases of circular, isotropic, and radial orbits, the maximum value of the term involving the velocity dispersions is $0,1 / 3$, and 1 , respectively. We use $1 / 3$ to be consistent with the $f_{\mathrm{PM}}$ value adopted for an isotropic orbit distribution.

For estimating $\rho$, here we assume an NFW mass profile and fit it to the mass profile. Since the NFW best-fit parameters are derived by fitting the uncorrected mass profile, we repeat the fitting using the corrected mass profile. This process is iterated where in each step we take the parameters of the most recent corrected mass profile. After a few iterations the mass profile converges (when we adopt $10^{-6}$ tolerance).

Error analysis. We assess the virial and projected mass uncertainty using the Jackknife technique, which was introduced by Quenouille (1949) and Tukey (1958). This is one of the simplest, widespread, and quickest resampling method techniques. The mass uncertainty is

$$
\begin{aligned}
d M_{\mathrm{v} / \text { proj }}(\leqslant r) & \approx d M(\leqslant R) \\
& =\frac{N-1}{N} \sqrt{\sum_{i=1}^{N}\left[M_{-i}(\leqslant R)-\bar{M}_{-i}(\leqslant R)\right]^{2}},
\end{aligned}
$$

\footnotetext{
34 The projected mass profile estimator is based on the projected mass $q$, where $q \equiv v^{2} R / G$, but is not the projected mass (Bahcall \& Tremaine 1981).
} 
Table 3

Clusters' Centers, Redshifts, and Number of Members

\begin{tabular}{|c|c|c|c|c|c|c|c|c|c|}
\hline \multirow[b]{2}{*}{ Cluster Name } & \multicolumn{2}{|c|}{ X-Ray Center (J2000) } & \multicolumn{2}{|c|}{ BCG Location (J2000) } & \multicolumn{2}{|c|}{ Galaxy Surface Number Density Peak Location } & \multirow[b]{2}{*}{$\begin{array}{c}z_{\mathrm{c}} \\
\text { (Cluster Members) }\end{array}$} & \multirow[b]{2}{*}{$\begin{array}{c}z_{\mathfrak{c}} \\
\text { (Gaussian Fit) }\end{array}$} & \multirow[b]{2}{*}{$N_{\text {gal }}$} \\
\hline & $\begin{array}{c}\text { R.A. } \\
\text { (hh:mm:ss (deg)) }\end{array}$ & $\begin{array}{c}\text { Decl. } \\
\text { (hh:mm:ss (deg)) }\end{array}$ & $\begin{array}{c}\text { R.A. } \\
\text { (dd:mm:ss (deg)) }\end{array}$ & $\begin{array}{c}\text { Decl. } \\
\text { (dd:mm:ss (deg)) }\end{array}$ & $\begin{array}{l}\text { R.A. } \\
\text { (deg) }\end{array}$ & $\begin{array}{l}\text { Decl. } \\
\text { (deg) }\end{array}$ & & & \\
\hline $\mathrm{A} 963$ & 10:17:03.74 (154.2656) & 39:02:49.2 (39.047) & 10:17:03.6 (154.265) & 39:02:49.42 (39.0471) & $154.2563 \pm 0.0117$ & $39.0500 \pm 0.0117$ & 0.204 & $0.2039 \pm 3 \times 10^{-4}$ & $202_{-49}^{+19}$ \\
\hline A2261 & $17: 22: 27.25(260.6135)$ & 32:07:58.6 (32.1329) & $17: 22: 27.20(260.6133)$ & 32:07:56.96 (32.1325) & $260.5820 \pm 0.0139$ & $32.0298 \pm 0.0139$ & 0.2251 & $0.225 \pm 3 \times 10^{-4}$ & $189_{-30}^{+21}$ \\
\hline A1423 & 11:57:17.26 (179.3219) & 33:36:37.4 (33.6104) & 11:57:17.37 (179.3224) & 33:36:39.5 (33.611) & $179.3047 \pm 0.0179$ & $33.6144 \pm 0.0179$ & 0.2141 & $0.2142 \pm 2 \times 10^{-4}$ & $257_{-29}^{+18}$ \\
\hline A611 & 08:00:56.83 (120.2368) & 36:03:24.1 (36.0567) & 08:00:56.82 (120.2368) & 36:03:23.58 (36.0565) & $120.2534 \pm 0.0155$ & $36.0763 \pm 0.0155$ & 0.2871 & $0.287 \pm 2 \times 10^{-4}$ & $244_{-39}^{+25}$ \\
\hline RXJ2129 & 21:29:39.94 (322.4164) & 00:05:18.8 (0.0886) & 21:29:39.96 (322.4165) & 00:05:21.16 (0.0892) & $322.4170 \pm 0.022$ & $0.0898 \pm 0.0220$ & 0.2339 & $0.2341 \pm 2 \times 10^{-4}$ & $305_{-27}^{+23}$ \\
\hline MACSJ1206 & 12:06:12.28 (181.5512) & $-08: 48: 02.4(-8.8007)$ & 12:06:12.15 (181.5506) & $-08: 48: 03.32(-8.8009)$ & $181.5380 \pm 0.0042$ & $-8.7953 \pm 0.0042$ & 0.4397 & $0.4391 \pm 3 \times 10^{-4}$ & $519_{-54}^{+43}$ \\
\hline CL2130 & $\cdots$ & $\cdots$ & 21:30:27 (322.6123) & $-00: 00: 24.48(-0.0068)$ & $322.6034 \pm 0.0192$ & $-0.0090 \pm 0.0192$ & 0.1361 & $0.1360 \pm 2 \times 10^{-4}$ & $337_{-15}^{+18}$ \\
\hline
\end{tabular}

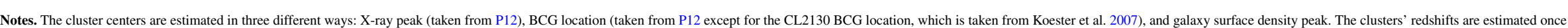
by taking the median of cluster members (see Appendix B.2), and once by a Gaussian fit to the galaxies' velocity histogram (see Section 3.1). The rightmost column is for the number of cluster members and infalling galaxies identified using the caustic method. 
where $M_{-i}(\leqslant R)$ is the mass estimation when we do not use the galaxy $i$ out of all galaxies within $R$, and $N$ is the number of galaxies inside $R$ (Efron 1981, and references within).

\subsubsection{Caustic Mass Estimator}

Diaferio \& Geller (1997) and D99 showed that the 3D mass profile can be estimated directly from the amplitude of the velocity caustics, $A(R)$ (for how to determine $A(R)$, see Appendix B.2.3),

$$
M_{\text {caustics }}(\leqslant r)=\frac{F_{\beta}}{G} \int_{0}^{r} A^{2}(R) d R,
$$

and its uncertainty ${ }^{35}$

$$
d M_{\text {caustics }}(\leqslant r)=\frac{2 F_{\beta}}{G} \int_{0}^{r} A(R) d A(R) d R .
$$

We adopt $F_{\beta}=0.7$ (for more details, see Appendix B.2.4).

\subsection{Halo Identification}

We are interested in estimating the contribution of mergers with different mass ratios to cluster growth. The first step is identifying the satellites' accreted and falling halos into the cluster. In Section 3.4.1, we present our scheme to identify these halos. Because our estimation depends on the identification scheme, in Section 3.4.2, we present a different scheme, the Friends of Friends (hereafter FoF), to identify these halos. The latter scheme will be used to test the sensitivity of our results to the halo finder (hereafter HF) used, and increase reliability.

In our case, the FoF scheme identifies more small and elongated mass halos. In addition, in some cases, one overdensity halo is identified as two closeby FoF halos. Nevertheless, using the two different schemes results in estimations which are in $1 \sigma$ agreement.

Since interlopers are already removed in a previous step (as described in Section 3.2), we only identify the halos using celestial coordinates and neglect line-of-sight separation within the halos.

\subsubsection{D Overdensity}

The method we present here is close in spirit to a combination of denMAX (Bertschinger \& Gelb 1991; Gelb \& Bertschinger 1994) and spherical overdensity (Lacey \& Cole 1994), which are two HFs commonly used in simulations. For each cluster, we make an initial velocity cut, as was described in Section 3.1, build a binary tree, as described in Appendix B.2, and identify galaxies that are bound to the cluster (cluster member galaxies and infalling galaxies) using the D99 procedure. The axes of the galaxy surface density map are cut to include all the identified bound galaxies and scaled with the same bin resolution, $B_{\text {res }}$. We then smoothed the galaxy surface density using a 2D Gaussian kernel with a fixed side size of $1 / 10$ (1/15 for MACSJ1206) of the identified galaxies' squared field of view. The amount of smoothing, $\sigma_{\text {kernel }}$, is taken to be equal in both axes, and $\sim 30 \%$ of the kernel size. This kernel size and level of smoothing are

\footnotetext{
35 By analyzing a sample of 3000 simulated clusters with masses of $M_{200}>10^{14} h_{0.73}^{-1} M_{\odot}$, Serra et al. (2011, hereafter S11) tested the deviation of this mass uncertainty recipe from the $1 \sigma$ confidence level. They found that on average at $r_{200}$ the upper mass uncertainty is underestimated by about $15 \%$ and the lower mass uncertainty is overestimated by about $25 \%$ (see right panel in their Figure 16; A. L. Serra 2012-2013, private communication).
}

optimized to include at least a few galaxies at the densest areas, but not to erase significant ( $3 \sigma$ above the average) features.

For identifying accreted halos (and infalling halos), after the smoothing we identify significant ( $3 \sigma$ above the average) galaxy surface density peaks. Now we need to estimate the halos' boundaries. As Kravtsov et al. (2004) mentioned, the virial radius is meaningless for the subhalos within a larger host as their outer layers are tidally stripped, and the extent of the halo is truncated. The definitions of the outer boundary of a subhalo and its mass are thus somewhat ambiguous. The truncation radius is commonly estimated at the point where $d \ln \rho(r) / d \ln r=-0.5$ since it is not expected that the density profile of the CDM halos will be flatter than this slope (Kravtsov et al. 2004). Kravtsov et al. (2004) note that this empirical definition of the truncation radius roughly corresponds to the radius at which the density of the gravitationally bound particles is equal to the background density of the host halo, albeit with a large scatter. In our case, although many of the halos are beyond the cluster virial radius, they are embedded in high-density regions with many galaxies in their surroundings. Estimating any specific characteristic radius, such as the truncation or the halo's virial radius, via a density profile is not possible because the data are not sufficient to estimate a density profile for all halos. Therefore, we follow the Kravtsov et al. suggestion and limit the halos to surface densities which are $2 \sigma$ above the average. Below this threshold, e.g., $\sim 1.5 \sigma$, the halos' sizes do not increase by much, and halos within the cluster virial radius are bridged to the cluster background.

If there are a few significant peaks inside one $2 \sigma$ region, we estimate the smoothed galaxy surface density minimum between them, i.e., $\Sigma_{\text {ridge. }}$ If $\Delta_{\text {peak }}=\Sigma_{\text {peak }} / \Sigma_{\text {ridge }}$, where $\Sigma_{\text {peak }}$ is the peak of the smoothed surface density, is smaller than some threshold (which is taken to be 1.1), we do not consider the peak with the lowest density to be significant. If $\Delta_{\text {peak }}$ is above the threshold, the $2 \sigma$ region is divided by a perpendicular (to the line connecting the two peaks) line which passes via the location of $\Sigma_{\text {ridge. }}$.

For each cluster, the surface density region which is $2 \sigma$ above the average and includes the cluster center (determined by the $\mathrm{X}$-ray peak) is notated as the cluster core and is not considered to be one of the accreted halos. The core region can be about the size of the cluster (as defined by the virial radius, as is the case for RXJ2129).

The uncertainties in the galaxies' surface density peaks for both the cluster and identified peaks are taken to be $0.5 B_{\text {res }} \sigma_{\text {kernel }}$. Different kernel sizes can of course shift the galaxies' surface density peaks, but they will be in agreement within $1 \sigma$.

\subsection{2. $2 D$ Friends of Friends (FoF)}

We adopt the widely used standard FoF algorithm (Huchra \& Geller 1982; Davis et al. 1985). Groups are defined by linking together all pairs of galaxies with separations less than some linking length, $l_{\text {linking }}$, which is taken to be some fraction, $b_{\text {frac }}$, of the averaged inter-galaxy spacing, $\bar{r}_{\text {inter }}$. The latter is estimated as the mean of all the distances between each galaxy and its closest neighbor. Thus, $l_{\text {linking }}=b_{\text {frac }} \bar{r}_{\text {inter }}$. Except for $b_{\text {frac }}$, the second free parameter is the minimum number of galaxies in a group, which we take to be $N_{\text {gal,min }}=6$ for consistency with the minimum number of galaxies in a halo found by the overdensity HF (see Table 6). Lower values for $N_{\text {gal,min }}$ increase the number of low-mass halos when many of them have a more filamentary shape than approximately round shape. Values of $b_{\text {frac }} \lesssim 0.45$ are too small to identify obvious high-mass halos, while values 
$b_{\text {frac }} \gtrsim 0.7$ are too large so halos are joined to the core. We set $b_{\text {frac }}=0.54$ (values in the $0.51-0.55$ range give almost identical results) for consistency with the overdensity HF. At $b_{\text {frac }}=0.5$ one of the A963 halos, which is identified by overdensity HF, is not identified by the FoF HF, and at $b_{\text {frac }}=0.56$ one of the A1423 halos, which is identified by overdensity HF, is fused with the core.

We consider only FoF halos that have most of their galaxies outside the cluster core (identified by the overdensity HF).

\subsection{Substructure and Relaxation Tests}

The mass estimators (virial and projected), which are used to estimate the halos' masses, assume that the halos are in steady state (see Section 3.3). Therefore, it is important to have an indication for the halos' relaxation levels. In addition, we are interested to see if there is a strong correlation between the infalling and accreted satellites' substructure and relaxation levels. In this section, we briefly describe the Dressler-Shectman test, which is used to measure the level of substructure, and center displacement tests, which are used to estimate the relaxation state.

\subsubsection{Dressler-Shectman Test}

In order to check for the presence of substructure in the 3D space, we compute the statistics devised by Dressler \& Shectman (1988, hereafter DS). Pinkney et al. (1996, hereafter P96) examined various substructure tests, and DS was found to be the most sensitive 3D test. The test works in the following way: for each galaxy that is a cluster member, the $N_{\text {local }}$ nearest neighbors are found, and the local velocity mean and dispersion are computed from this sample of $N_{\text {local }}+1$ galaxies. The deviation of the local velocity mean and dispersion from the cluster velocity mean and dispersion is calculated,

$$
\delta_{i}^{2}=\frac{N_{\text {local }}+1}{\sigma^{2}}\left[\left(\bar{v}_{\text {local }, i}-\bar{v}\right)^{2}+\left(\sigma_{\text {local }, i}-\sigma\right)^{2}\right],
$$

where $\bar{v}$ and $\sigma$ are the global dynamical parameters and $\bar{v}_{\text {local }, i}$ and $\sigma_{\text {local }, i}$ are the local mean velocity and velocity dispersion of galaxy $i$, determined using itself and its $N_{\text {local }}$ closest galaxies. Note that other forms of $\delta_{i}$ were also suggested (e.g., Biviano et al. 2002). DS also defined the cumulative deviation

$$
\Delta=\sum_{i=1}^{N_{\mathrm{gal}}} \delta_{i}
$$

where $N_{\text {gal }}$ is the number of cluster members.

It is necessary to calibrate the $\Delta$ statistics for each cluster (DS; Knebe \& Muller 2000). The calibration is done by randomly shuffling among the positions. If substructure exists, the shuffling erases any correlation between redshifts and positions. We have done this procedure $10^{4}$ times for each cluster. Then, we define $P\left(\Delta_{\mathrm{s}}>\Delta_{\text {obs }}\right)$ as the fraction of the total number of Monte Carlo models of the cluster that have shuffled values, $\Delta_{\mathrm{s}}$, larger than the cluster observed value, $\Delta_{\text {obs. }} P\left(\Delta_{\mathrm{s}}>\Delta_{\text {obs }}\right) \sim 1$ means that the cluster contains no substructure, while $P\left(\Delta_{\mathrm{s}}>\Delta_{\text {obs }}\right) \sim 0$ indicates that the cluster contains statistically significant substructure.

Originally, DS proposed the computation of $\Delta$ using $N_{\text {local }}=$ 10 independently of the number of galaxy cluster members. Bird (1994, and references therein) pointed out that using a constant value for the number of nearest neighbors reduces the sensitivity of this test to significant structures, and suggested using $N_{\text {local }}=\sqrt{N_{\text {gal }}}$.

The test is sensitive to outliers (P96), and therefore we use it also to identify which interloper removal method is more adequate to deal with outliers (see Section 6).

\subsubsection{Relaxation Tests}

The distinction between relaxed and unrelaxed clusters is made by estimating the displacement between two different definitions for the cluster center. These relaxation proxies have been widely used with both data (e.g., P12) and simulations (e.g., Neto et al. 2007; Macciò et al. 2007; Lemze et al. 2012). Sometimes they are also interpreted as a measure of the substructure level (see Thomas et al. 2001).

We used two different displacements. The first test is the displacement between the galaxy surface density peak, $\mathbf{R}_{\mathrm{sd}}$, and the potential center (potential minimum), $\mathbf{R}_{\mathrm{p}}$, which is estimated as follows:

$$
\Phi\left(\mathbf{R}_{\mathrm{p}}\right)=\min \left\{-G M_{\mathrm{gal}} \sum_{i=1}^{N_{\mathrm{gal}}} \frac{1}{\left|\mathbf{R}_{\mathrm{i}}-\mathbf{R}\right|}\right\},
$$

where $M_{\text {gal }}$ is taken to be $10^{12} h_{0.73}^{-1} M_{\odot}$, the value used to build the binary tree (see Appendix B.2). The potential center is calculated using galaxies within the halo's boundaries, which are taken to be the caustic virial radius for the clusters and the $2 \sigma$ contours for the infalling and accreted satellites (for more details, see Section 5.3). The displacement is normalized with respect to a scale radius, $r_{\text {scale, }}$, which is the caustic virial radius for the clusters, and an effective radius, $r_{\text {eff }}=\sqrt{S} / \pi$, for the halos, where $S$ is the area inside the $2 \sigma$ contour

$$
r_{\mathrm{sp}}=\left|\mathbf{R}_{\mathrm{sd}}-\mathbf{R}_{\mathrm{p}}\right| / r_{\text {scale }} .
$$

The second test is the displacement between the center of mass, $\mathbf{R}_{\mathrm{cm}}$, and the potential center

$$
r_{\mathrm{cp}}=\left|\mathbf{R}_{\mathrm{cm}}-\mathbf{R}_{\mathrm{p}}\right| / r_{\text {scale }} .
$$

The center of mass is estimated by taking the galaxies' projected locations and assuming that the DM particles are distributed like the galaxies.

In equilibrium, $r_{\mathrm{sp}}$ and $r_{\mathrm{cp}}$ are expected to vanish, while high values, $\gtrsim 1$, indicate an unrelaxed halo. The threshold between the two phases is quite arbitrary. Note also that due to projections these criteria are less sensitive to LOS mergers.

\section{EXPECTED FRACTION OF CLUSTER MASS ACCRETION}

Here we estimate the expected fraction of cluster mass accretion by following the merger rates and mass assembly histories of DM halos in the two Millennium simulations (FMB10; G10).

The halo's mass as a function of redshift can be fitted-using a two parameter $\left(\beta_{\mathrm{MAH}}\right.$ and $\left.\gamma_{\mathrm{MAH}}\right)$ function

$$
M\left(M_{0}, z\right)=M_{0}(1+z)^{\beta_{\mathrm{MAH}}\left(M_{0}\right)} \exp \left(-\gamma_{\mathrm{MAH}}\left(M_{0}\right) z\right)
$$

where $M_{0}=M(z=0)$ and can be expressed as a function of the observed cluster mass, $M_{\mathrm{obs}}$, and observed redshift, $z_{\mathrm{obs}}$,

$$
M_{0}=\frac{M_{\mathrm{obs}}}{\left(1+z_{\mathrm{obs}}\right)^{\beta_{\mathrm{MAH}}\left(M_{0}\right)} \exp \left(-\gamma_{\mathrm{MAH}}\left(M_{0}\right) z_{\mathrm{obs}}\right)} .
$$


This function is versatile enough to accurately capture the main features of most mass accretion histories (MAHs) in the Millennium simulation (McBride et al. 2009; see also Tasitsiomi et al. 2004). The parameters in Equation (13) are derived by fitting this function to the mass history inferred from the mean mass growth rates of halos (see Equation (2) in FMB10)

$$
\langle\dot{M}\rangle=46.1 M_{\odot} \mathrm{yr}^{-1}\left(\frac{M(z)}{h_{0.73}^{-1} 10^{12} M_{\odot}}\right)^{1.1}(1+1.11 z) \epsilon(z),
$$

where $\epsilon(z)$ is the normalized Hubble function, i.e., $\epsilon(z)=$ $\sqrt{\left(\Omega_{\mathrm{m}}(1+z)^{3}+\Omega_{\Lambda}\right)}$. For $\beta_{\mathrm{MAH}}$ and $\gamma_{\mathrm{MAH}}$ explicit dependence on $M_{0}$, see Appendix D.

FM08 introduced merger rates that fit well the ones found in the Millennium simulations I and II (G10; FMB10). The mean merger rate of halos with the descendant mass $M$ with other halos with mass ratio $\zeta$ at $z$ in units of mergers per halo per unit redshift per unit $\zeta$ is

$$
\begin{aligned}
\frac{d N_{\mathrm{m}}}{d \zeta d z}\left(M_{0}, \zeta, z\right)= & A_{\mathrm{m}}\left(\frac{M\left(M_{0}, z\right)}{h_{0.73}^{-1} 10^{12} M_{\odot}}\right)^{\alpha} \zeta^{\beta} \\
& \times \exp \left(\left(\frac{\zeta}{\tilde{\zeta}}\right)^{\gamma}\right)(1+z)^{\eta},
\end{aligned}
$$

when $A_{\mathrm{m}}=0.065, \alpha=0.15, \beta=-1.7, \gamma=0.5, \tilde{\zeta}=0.4$, and $\eta=0.0993$. These (especially $\beta, \gamma, \tilde{\zeta}$, and $A_{\mathrm{m}}$ ) values are obtained by taking special care of halo fragmentation and ensuring that the mass contribution of each merger to halo growth is counted just once (G10). There is some uncertainty in the value of $\alpha$. FMB10 found a lower value of $\alpha(\alpha=0.133)$. We show the expected fraction of cluster mass accretion assuming 0.133 as well.

The Millennium simulation has a low number of massive cluster-sized halos; therefore, the statistics in this mass regime is limited. Wu et al. (2013) analyzed a sample of 96 halos in the $10^{14.8 \pm 0.05} h^{-1} M_{\odot}$ mass range (about 4 times the number of halos with similar mass in the Millennium simulation) from the Rhapsody cluster re-simulation project. They found that the number of mergers per halo per unit redshift per unit $\zeta$ is consistent in $1 \sigma$ with the one found in the Millennium simulation (see their Figure 6, right panel).

The mean mass accumulation per halo per unit redshift per unit $\zeta$ is

$$
M_{\mathrm{acc}}\left(M_{0}, \zeta, z\right)=\frac{d N_{\mathrm{m}}}{d \zeta d z} M_{\text {small }},
$$

where $M_{\text {small }}$ is the mass of the less massive progenitor of each merger (G10). Since $M=M_{\mathrm{mp}}+M_{\mathrm{small}}$, where $M_{\mathrm{mp}}$ is the main progenitor mass, and $\zeta \equiv M_{\mathrm{small}} / M_{\mathrm{mp}}$,

$$
M_{\text {small }}\left(M_{0}, \zeta, z\right)=M\left(M_{0}, z\right) \frac{\zeta}{1+\zeta} .
$$

Thus, the fraction of the cluster mass at $z=z_{1}$ accumulated at $z_{1} \leqslant z \leqslant z_{2}$ by progenitors with mass ratios $\zeta_{1} \leqslant \zeta \leqslant \zeta_{2}$ is

$$
F\left(M_{\mathrm{obs}}, z_{\mathrm{obs}}\right)=\frac{1}{M\left(z_{1}\right)} \int_{\zeta_{1}}^{\zeta_{2}} d \zeta \int_{z_{1}}^{z_{2}} d z M_{\mathrm{acc}}\left(M_{0}, \zeta, z\right) .
$$

We compare these predicted values to the ones based on observations. For the comparison, we took $z_{2}=z_{\text {obs }}$ and $z_{1}=z_{\mathrm{f}}$, which is the redshift when all the bound matter falls onto the cluster (for more details, see Section 4.2).

\subsection{Correcting $\sigma_{8}$}

In the Millennium simulations I (Springel et al. 2005) and II (Boylan-Kolchin et al. 2009), the linear mass density fluctuation amplitude in an $8 h_{0.73}^{-1} \mathrm{Mpc}$ sphere at redshift zero, $\sigma_{8}$, is 0.9 , while the latest value is 0.82 (Bennett et al. 2013; Hinshaw et al. 2013). A lower value for $\sigma_{8}$ means slower structure formation, and therefore a lower $\sigma_{8}$ is equivalent to a higher redshift in a high $\sigma_{8}$ universe. More specifically, we follow Angulo et al. (2012) and correct the expectations derived from the Millennium simulations, which are based on the values found by analyzing WMAP1 (the first-year data from the Wilkinson Microwave Anisotropy Probe (WMAP) satellite; Spergel et al. 2003 ), to the ones based on the values found by analyzing WMAP7 (Komatsu et al. 2011), which are more updated and closer to the latest value. Following Angulo et al. (2012), we denote the Millennium $\left(\Omega_{\mathrm{m}}=0.25\right.$ and $\left.\sigma_{8}=0.9\right)$ and WMAP7 $\left(\Omega_{\mathrm{m}}=0.272\right.$ and $\sigma_{8}=0.807$; Komatsu et al. 2011) cosmologies by $A$ and $B$, respectively. Angulo $\&$ White (2010) suggested scaling the Millennium simulations' final redshift, $z_{\mathrm{A}}^{f}$ (so that $z_{\mathrm{A}} \geqslant z_{\mathrm{A}}^{f}$ ), and box size scale factor, $s$ (so that $L_{\mathrm{A}}=s L_{\mathrm{B}}$ ), to keep the linear fluctuation amplitude the same in the two cosmologies, which is equivalent to having the same halo mass function derived in Press-Schechter theory. For a zero final redshift in the WMAP7 cosmology, $z_{\mathrm{B}}^{f}=0$, they found $z_{\mathrm{A}}^{f}=0.319$ and $s=1.072$ (Angulo et al. 2012).

To find the target redshifts, $z_{\mathrm{A}}$, we follow Angulo \& White (2010, their Equation (5)):

$$
D_{A}\left(z_{\mathrm{A}}\right)=D_{B}\left(z_{\mathrm{B}}\right) \frac{D_{A}\left(z_{\mathrm{A}}^{f}\right)}{D_{B}\left(z_{\mathrm{B}}^{f}\right)},
$$

where $D$ is the linear growth factor,

$$
D(z)=D_{0} \epsilon(z) \int_{0}^{a(z)} \frac{1}{a^{3} \epsilon(a)^{3}} d a,
$$

where $a=(1+z)^{-1}$ is the scale factor and $D_{0}$ is a constant set by the normalization $D(0)=1$. This redshift transformation is applied to $z_{\mathrm{obs}}, z_{1}$, and $z_{2}$.

After scaling the positions with $s$, to keep the mass density the same in both cosmologies, the particle mass is scaled as follows: ${ }^{36}$

$$
m_{\mathrm{p}, \mathrm{A}}=\frac{\Omega_{\mathrm{m}, \mathrm{A}} h_{\mathrm{A}}^{2} L_{\mathrm{A}}^{3}}{\Omega_{\mathrm{m}, \mathrm{B}} h_{\mathrm{B}}^{2} L_{\mathrm{B}}^{3}} m_{\mathrm{p}, \mathrm{B}},
$$

where in our case $h_{\mathrm{A}}=h_{\mathrm{B}}=0.73$ (Angulo \& White 2010). Since the halos at the two different cosmologies have the same particle number, the difference in their mass is only due to the scaling of the particle mass. Thus,

$$
M_{\mathrm{obs}, \mathrm{A}}=\frac{\Omega_{\mathrm{m}, \mathrm{A}}}{\Omega_{\mathrm{m}, \mathrm{B}}} s^{3} M_{\mathrm{obs}, \mathrm{B}} .
$$

In this case, the difference in $M_{\mathrm{obs}}$ due to the different cosmologies is negligible, but $M_{\mathrm{obs}}$ was still scaled for correctness.

\subsection{Estimating $z_{f}$}

We are interested in comparing our simulation-based expectations of clusters' mass growth with our estimated ones. Therefore, in our expectation calculations (Equation (19)) we

\footnotetext{
36 The power of $h$ is 2 and not 3 because $m_{\mathrm{p}} \propto h^{-1}$.
} 
need to take the same redshift range as the observed one. For each cluster, the observed redshift range of the clusters' mass accumulation starts at about the cluster's redshift, $z_{2}=z_{\mathrm{obs}}=z_{\mathrm{c}}$. For simplicity, we neglect the fact that accreted satellites are partly or fully within the virial radius, and so $z_{2}$ is actually higher than $z_{\mathrm{c}}$ (which increases the expected $F$ ). The observed redshift range ends at the redshift when all the infalling matter reaches the cluster's virial radius, $z_{1}=z_{\mathrm{f}}$. Below we explain how we estimated $z_{\mathrm{f}}$.

For each cluster, we estimate how much time it takes for matter to fall from the furthest radius where galaxies are bound and falling onto the cluster, $r_{\max }$ (for more details, see Section 4.3), to the clusters' virial radius, $r_{\text {caustics, vir. We do }}$ that by first separating this radial range into $N_{\text {sections }}$ smaller sections, corresponding to $\Delta r=\left(r_{\max }-r_{\text {caustics, vir }}\right) / N_{\text {sections }}$. We take $N_{\text {sections }}=100$, when larger values do not change the result significantly. The infalling time in section $i$ is $\Delta t\left(r_{i}\right)=\left(-v_{0}\left(r_{i}\right)+\sqrt{v_{0}\left(r_{i}\right)^{2}+2 a_{\mathrm{r}}\left(r_{i}\right) \Delta r}\right) / a_{\mathrm{r}}\left(r_{i}\right)$, where $a_{\mathrm{r}}(r)=$ $G M_{\text {caustics,vir }} / r^{2}$ and $v_{0}$ is the velocity at the beginning of the section, which propagates as $v_{0}\left(r_{i+1}\right)=a_{\mathrm{r}}\left(r_{i}\right) \Delta t\left(r_{i}\right)+v_{0}\left(r_{i}\right)$. Then we sum the time of all sections, $t=\sum_{i} \Delta t_{i}$. Finally, we convert the time into redshift, $t=H_{0}^{-1} \int_{z_{\mathrm{c}}}^{z_{\mathrm{f}}} d z 1 /(a(z) \epsilon(z))$, to yield $z_{\mathrm{f}}$. Because the cluster's mass depends on the redshift (see Equation (13)), for each cluster we iterate this process. In each iteration, we determined the cluster's final mass by the final redshifts inferred in the previous iteration. The process stops when the final cluster's mass converges (when we adopt $10^{-4}$ tolerance).

\subsection{Estimating $r_{\max }$}

We follow Rines et al. (2013) and estimate the furthest radius where galaxies are bound and falling onto the cluster, $r_{\max }$, in a conservative manner,

$$
r_{\max }=\min \left(r_{\text {caustics, } \max }, r_{\text {bound,max }}\right) \text {, }
$$

where $r_{\text {caustics, max }}$ is the maximum extent of the caustics (the maximum radius where the caustics are above zero) and $r_{\text {bound,max }}$ is the maximum radius where all the galaxies are bound. The latter is estimated to be the radius where $\bar{\rho}\left(<r_{\text {bound }}\right)=\Delta_{c} \rho_{\text {crit }}$, where $\rho_{\text {crit }}$ is the critical density of the universe at the cluster redshift. The final overdensity to the critical density at collapse, $\Delta_{c}$, can be derived from the critical mean overdensity interior to the last shell that will collapse in the future, $\delta_{c}$,

$$
\Delta_{c}=\Omega_{\mathrm{m}}\left(\delta_{c}+1\right)
$$

since the critical (final) cluster mean density contrast is $\delta_{c}=$ $\left(\bar{\rho}-\rho_{\mathrm{m}}\right) / \rho_{\mathrm{m}}$ and $\rho_{\mathrm{m}} \equiv \Omega_{\mathrm{m}} \rho_{\text {crit }}$.

For calculating $\delta_{c}$, we use the expressions presented in Lokas $\&$ Hoffman (2001), who used the formalism of spherical tophat collapse,

$$
\delta_{c}(z)=\frac{1}{\Omega_{\mathrm{m}}(z)} u(z)-1,
$$

(their Equation (22)), where $u(z)=1+5 \Omega_{\Lambda}(z) / 4+3 \Omega_{\Lambda}(z)(8+$ $\left.\Omega_{\Lambda}(z)\right) /(4 v(z))+3 v(z) / 4$ (their Equation (23)) and $v(z)=$ $\left(\Omega_{\Lambda}(z)\left(8-\Omega_{\Lambda}(z)^{2}+20 \Omega_{\Lambda}(z)+8\left(1-\Omega_{\Lambda}(z)\right)^{3 / 2}\right)\right)^{1 / 3}$ (their Equation (24)), where $\Omega_{\mathrm{m}}(z)$ and $\Omega_{\Lambda}(z)$ are the ratio of the matter and dark energy densities to the critical density, respectively.

Nagamine \& Loeb (2003) showed that although Lokas \& Hoffman (2001) have ignored the possibility that the mass shell may have a nonzero initial peculiar velocity, for $\Omega_{\mathrm{m}}(0)=0.3$, $\Omega_{\Lambda}(0)=0.7$, and initial time as $z=0$, on average, the analytic estimation for $\delta_{c}$ based on the spherical tophat collapse model appears to provide a good approximation to the actual threshold (see also Busha et al. 2003). Dünner et al. (2006) showed that, on average, about $10 \%$ of the mass enclosed by $r_{\text {bound,max }}$ is not bound, while about the same percentage is bound mass that lays beyond this radius. Therefore, they claimed that this radius encloses as much mass as will remain bound to the distant future (leaving about as many bound galaxies outside as unbound ones inside).

\subsection{The Bias in the $\zeta$ and $F$ Values Estimated Using $N$-body Simulations due to the Exclusion of Baryons}

The Millennium simulations include only DM particles, while the halo mass estimated from the data using galaxy dynamics is of the total matter. If the baryon fraction, $f_{\mathrm{b}}$, is independent of the halo mass, mass ratio estimations using only the DM are identical to the ones of total matter. However, this is not the case, and the baryon fraction depends on halo mass because in halos with lower mass baryons more easily escape the halo gravitational potential well (Lin et al. 2003; Giodini et al. 2009; Dai et al. 2010, though see also Gonzalez et al. 2007, who found that $f_{\mathrm{b}}$ is independent of the halo mass).

Here we roughly evaluate the bias in the $\zeta$ and $F$ values estimated using $N$-body simulations (such as the Millennium simulations) due to the exclusion of baryons. Generally, highmass halos have a higher baryon fraction. Therefore, $\zeta$ and $F$ predictions based on $N$-body simulations are expected to decrease (they are biased upward) when taking baryons into consideration.

Dai et al. (2010) assumed that all the gas pressure is thermal and estimated the baryon fraction dependence on the X-ray temperature, $T_{\mathrm{X}}$, as

$$
\log f_{\mathrm{b}}=(-1 \pm 0.02)+(0.2 \pm 0.03) \log T_{\mathrm{X}}
$$

at $1 \mathrm{keV} \lesssim T_{\mathrm{X}} \lesssim 10 \mathrm{keV}$. In order to convert the temperature into the halo mass, we use the mass-temperature relation derived by Wojtak \& Łokas (2010),

$$
M_{\mathrm{vir}}=(7.85 \pm 0.55)\left[T_{\mathrm{X}} /(5 \mathrm{keV})\right]^{1.54 \pm 0.12} 10^{14} h_{0.7}^{-1} M_{\odot} .
$$

This relation was derived from 23 nearby $(z<0.1)$ relaxed galaxy clusters whose masses were estimated by kinematic data, and whose $T_{\mathrm{X}}$ were taken from Horner (2001). The temperature range mentioned above is equivalent to the $6.6 \times 10^{13} \lesssim M_{\text {vir }} \lesssim$ $2.3 \times 10^{15} h_{0.7}^{-1} M_{\odot}$ mass range. Although not all of our infalling and accreted halos are relaxed, nor are their masses defined to be the virial one, we use these expressions to roughly estimate the effect of including baryons to the $\zeta$ and $F$ values estimated from the Millennium simulations.

Since $M_{\mathrm{tot}} \equiv M_{\mathrm{DM}}+M_{\mathrm{b}}$, where $M_{\mathrm{b}}$ is the baryon mass, and $M_{\mathrm{b}} \equiv M_{\text {tot }} f_{\mathrm{b}}\left(M_{\mathrm{tot}}\right)$, we get

$$
M_{\mathrm{DM}}=M_{\mathrm{tot}}\left(1-f_{\mathrm{b}}\left(M_{\mathrm{tot}}\right)\right) .
$$

The corrected mass ratio is therefore

$$
\zeta \equiv \frac{M_{\mathrm{tot}, \mathrm{small}}}{M_{\mathrm{tot}, \mathrm{mp}}}=\frac{M_{\mathrm{DM}, \mathrm{small}}}{M_{\mathrm{DM}, \mathrm{mp}}} \times \mathrm{f}_{\mathrm{corr}}\left(M_{\mathrm{DM}, \mathrm{small}}, M_{\mathrm{DM}, \mathrm{mp}}\right),
$$

where the correction factor is

$$
\mathrm{f}_{\mathrm{corr}}\left(M_{\mathrm{DM}, \mathrm{small}}, M_{\mathrm{DM}, \mathrm{mp}}\right)=\frac{1-f_{\mathrm{b}}\left(M_{\mathrm{tot}, \mathrm{mp}}\left(M_{\mathrm{DM}, \mathrm{mp}}\right)\right)}{1-f_{\mathrm{b}}\left(M_{\mathrm{tot}, \mathrm{small}}\left(M_{\mathrm{DM}, \mathrm{small}}\right)\right)} \text {. }
$$


For correcting $F$, we need to insert the denominator of the correction factor into the integration over $\zeta$ in Equation (19). However, a first-order correction for a narrow $\zeta$ range is to multiply $F$ by $\mathrm{f}_{\text {corr }}$. We estimate the correction for our lowest mass ratio bin, $\zeta \sim 0.1$, where the correction is the largest. We take $M_{\text {tot,mp }}$ to be $M_{\text {caustics,vir }}$, which on average is $8 \times 10^{14} h_{0.73}^{-1} M_{\odot}$, so for $\zeta \sim 0.1, M_{\text {tot } \text {,small }}=8 \times 10^{13} h_{0.73}^{-1} M_{\odot}$. Then, we convert the masses to $T_{\mathrm{X}}$ using Equation (28) and convert the $T_{\mathrm{X}}$ to $f_{\mathrm{b}}$ using Equation (27) to yield $f_{\text {corr }}=0.96$. This $4 \%$ correction is much smaller than our uncertainties.

\section{RESULTS}

In this section, we present our results for the first portion of CLASH clusters: A963, A2261, A1423, RXJ2129, A611, MACSJ1206, and CL2130, which is in the foreground of the cluster RXJ2129. Table 3 gives information about the clusters' centers, redshifts, and number of cluster members identified by the D99 method (for more details see Appendix B.2), which also includes infalling matter. The cluster centers are estimated in three different ways: X-ray peak (taken from P12), BCG location (taken from P12, except for the CL2130 BCG location, which is taken from Koester et al. 2007), and galaxy surface density peak. For all clusters, the X-ray peaks and the BCG locations are in agreement within 3 arcsec (except for CL2130, where we do not have an X-ray peak). The X-ray peaks and the galaxy surface density peaks are also in agreement within $\sim 1 \sigma$ for all clusters, except MACSJ1206, where it is $\sim 2.5 \sigma$, and A2261, where the galaxy surface density peak is at a halo fused below the cluster core (see our definition for the cluster core at Section 3.4.1).

The clusters' redshifts are estimated once by taking the median of cluster members (see Appendix B.2), and once by a Gaussian fit to the galaxies' velocity histogram (see Section 3.1). We find that both redshift estimations are in agreement within $10^{-3}$ accuracy (see Table 3 ). This agreement is when each cluster has $\sim$ a few hundred cluster members. At lower numbers of galaxies, the Gaussian fit uncertainty is higher, and the D99 method is not very reliable when there are less than $\sim 100$ cluster members (M. Geller 2012, private communication). D99 mentioned that clusters may have multiple X-ray peaks. Thus, he suggested defining the center of the cluster as the galaxy surface density peak. However, all of the clusters in our sample have a single $\mathrm{X}$-ray peak, and since its uncertainty is smaller (and in agreement with the BCG location), we choose it to be the cluster center.

\subsection{Clusters' Mass Profiles}

The clusters' mass profiles are shown in Figure 1. For each cluster, the caustic (black curves), virial (blue curves), projected (red curves), and X-ray (green curves) mass profiles are estimated. For each of these profiles, the solid curve represents the mean value and the dashed curves the $\pm 1 \sigma$ uncertainty. For convenience, we zoom in on the 2000-5000 $h_{0.73}^{-1} \mathrm{kpc}$ radius range of the mass profiles of each cluster. For consistency, all dynamical mass profiles are estimated after using the same interloper removal method. Since we estimate the caustic mass profile, it is natural to use the caustic interloper removal method.

The virial radius is estimated to be the radius where $\bar{\rho}(<r)=$ $\Delta_{c} \rho_{\text {crit }}$, where $\rho_{\text {crit }}$ is the critical density of the universe at the cluster redshift. The final overdensity to the critical density

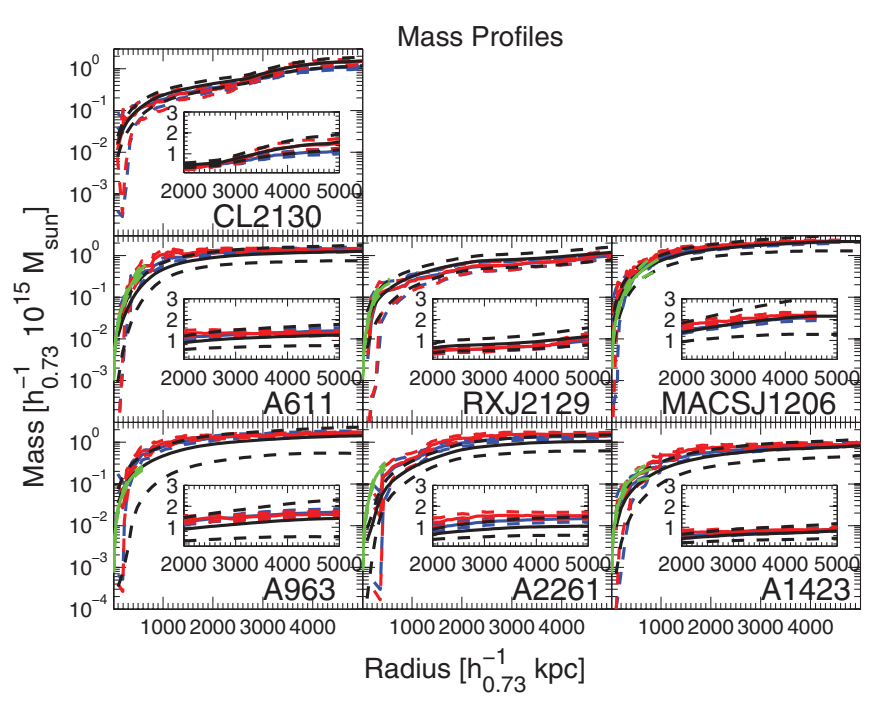

Figure 1. Mass profiles. The caustic (black curves), virial (blue curves), projected (red curves), and X-ray (green curves) mass profiles are estimated. For all the mass estimators, the solid curve represents the mean value and the dashed curves the $\pm 1 \sigma$. The caustic mass profile is extrapolated to radii $\lesssim 200$ $h_{0.73}^{-1} \mathrm{kpc}$. In a few cases and mainly at small radii, the projected and virial mass profiles decrease with an increasing radius. The mass estimation at these radii is based on a low number of galaxies (3-6), so the uncertainty is large. In any case, the decrease is negligible comparing with the uncertainty at these radii. For convenience, we zoom in on the 2000-5000 $h_{0.73}^{-1} \mathrm{kpc}$ radius range of the mass profiles of each cluster.

(A color version of this figure is available in the online journal.)

at collapse is taken to be $\Delta_{c}=18 \pi^{2}+82 x-39 x^{2}$, where $x \equiv \Omega_{m}(z)-1$ (Bryan \& Norman 1998). For estimating $r_{200}$, which is the radius where the final overdensity to the critical density at collapse is 200 , we take $\Delta_{c}=200$. Then we estimate $M_{\text {vir }}$ and $M_{200}$ masses by $M_{\text {vir }}=(4 \pi / 3) \Delta_{c} \rho_{\text {crit }} r_{\text {vir }}^{3}$ and $M_{200}=(4 \pi / 3) 200 \rho_{\text {crit }} r_{200}^{3}$, respectively. For estimating the $r_{200}, r_{\mathrm{vir}}, M_{200}$, and $M_{\mathrm{vir}}$ uncertainties, we make $10^{4}$ realizations for each of the clusters' mass profiles. In each realization and for each mass bin, we randomly take a value from a Gaussian distribution. The Gaussian mean and standard deviation are taken to be the bin's mass and its uncertainty, respectively. Finally, we estimate the $r_{200}, r_{\mathrm{vir}}, M_{200}$, and $M_{\mathrm{vir}}$ uncertainties as the standard deviations of all these realizations. In Table 4, we present our estimations for these uncertainties for two of the dynamical mass profiles, caustics and projected (the virial mass profiles are quite similar to the projected mass profiles; see Figure 1). The last column in Table 4 is the uncorrected virial mass estimated using the projected mass estimator, $M_{\text {proj,vir }}$, which is needed in Section 5.3.

Note that $M_{\mathrm{vir}}$ is the cluster's mass within a sphere with an average density of $\Delta_{c}$ times the critical one, not to be confused with the virial mass profile, $M_{\mathrm{v}}(r)$ (see Section 3.3.1).

\subsection{Clusters' Dynamical States}

In this section, we estimate the clusters' dynamical states using the proxies described in Section 3.5. In Table 5, we present our substructure level estimations using the DS test for two different values of $N_{\text {local }}, 10$ and $\sqrt{N_{\text {gal }}}$, and after cleaning the interlopers with the two different procedures, HK96 and D99. The DS test is estimated considering only galaxies within the caustic virial radius. We also estimate the clusters' relaxation state using cluster center displacements (see Section 3.5.2) after cleaning interlopers using the D99 method. 
Table 4

Estimations for the Clusters' $r_{200}, r_{\mathrm{vir}}, M_{200}, M_{\mathrm{vir}}$ Using Two Different Mass Estimators: Caustics and Projected

\begin{tabular}{|c|c|c|c|c|c|c|c|c|c|}
\hline \multirow[b]{2}{*}{ Cluster Name } & \multicolumn{4}{|c|}{ Caustics } & \multicolumn{5}{|c|}{ Projected } \\
\hline & $\begin{array}{l}r_{\text {caustics, } 200} \\
\left(h_{0.73}^{-1} \mathrm{kpc}\right)\end{array}$ & $\begin{array}{c}r_{\text {caustics, vir }} \\
\left(h_{0.73}^{-1} \mathrm{kpc}\right)\end{array}$ & $\begin{array}{c}M_{\text {caustics, } 200} \\
\left(10^{15} h_{0.73}^{-1} M_{\odot}\right)\end{array}$ & $\begin{array}{c}M_{\text {caustics, vir }} \\
\left(10^{15} h_{0.73}^{-1} M_{\odot}\right)\end{array}$ & $\begin{array}{c}r_{\text {Cproj, } 200} \\
\left(h_{0.73}^{-1} \mathrm{kpc}\right) \\
\end{array}$ & $\begin{array}{c}r_{\text {Cproj, vir }} \\
\left(h_{0.73}^{-1} \mathrm{kpc}\right) \\
\end{array}$ & $\begin{array}{c}M_{\mathrm{Cproj}, 200} \\
\left(10^{15} h_{0.73}^{-1} M_{\odot}\right)\end{array}$ & $\begin{array}{c}M_{\text {Cproj,vir }} \\
\left(10^{15} h_{0.73}^{-1} M_{\odot}\right)\end{array}$ & $\begin{array}{c}M_{\text {proj,vir }} \\
\left(10^{15} h_{0.73}^{-1} M_{\odot}\right)\end{array}$ \\
\hline A963 & $1763 \pm 333$ & $2255 \pm 407$ & $0.82 \pm 0.48$ & $0.97 \pm 0.54$ & $2092 \pm 75$ & $2574 \pm 75$ & $1.36 \pm 0.13$ & $1.45 \pm 0.11$ & $1.76 \pm 0.13$ \\
\hline A2261 & $1361 \pm 251$ & $1935 \pm 320$ & $0.38 \pm 0.23$ & $0.64 \pm 0.29$ & $2016 \pm 249$ & $2538 \pm 196$ & $1.25 \pm 0.34$ & $1.43 \pm 0.25$ & $2.01 \pm 0.19$ \\
\hline A1423 & $1234 \pm 229$ & $1648 \pm 289$ & $0.28 \pm 0.16$ & $0.39 \pm 0.20$ & $1660 \pm 162$ & $2060 \pm 147$ & $0.69 \pm 0.16$ & $0.75 \pm 0.13$ & $0.94 \pm 0.10$ \\
\hline A611 & $1701 \pm 259$ & $2120 \pm 298$ & $0.80 \pm 0.32$ & $0.93 \pm 0.35$ & $2013 \pm 91$ & $2396 \pm 95$ & $1.32 \pm 0.15$ & $1.34 \pm 0.14$ & $1.59 \pm 0.11$ \\
\hline RXJ2129 & $1446 \pm 225$ & $1904 \pm 279$ & $0.46 \pm 0.21$ & $0.61 \pm 0.25$ & $1230 \pm 182$ & $1553 \pm 207$ & $0.29 \pm 0.12$ & $0.33 \pm 0.14$ & $1.02 \pm 0.16$ \\
\hline MACSJ1206 & $1916 \pm 225$ & $2303 \pm 268$ & $1.34 \pm 0.45$ & $1.52 \pm 0.51$ & $2090 \pm 63$ & $2462 \pm 59$ & $1.74 \pm 0.15$ & $1.85 \pm 0.13$ & $2.31 \pm 0.13$ \\
\hline CL2130 & $1282 \pm 149$ & $1716 \pm 182$ & $0.29 \pm 0.10$ & $0.38 \pm 0.11$ & $1144 \pm 157$ & $1518 \pm 203$ & $0.21 \pm 0.08$ & $0.26 \pm 0.09$ & $0.71 \pm 0.10$ \\
\hline
\end{tabular}

Table 5

Clusters' Substructure Levels and Relaxation Degrees

\begin{tabular}{|c|c|c|c|c|c|c|}
\hline \multirow[b]{2}{*}{ Cluster name } & \multicolumn{4}{|c|}{ D99 } & \multicolumn{2}{|c|}{ HK96 } \\
\hline & $\begin{array}{c}\mathrm{DS}\left(N_{\text {local }}=10\right) \\
P\left(\Delta_{\mathrm{S}}>\Delta_{\mathrm{obs}}\right)(\%)\end{array}$ & $\begin{array}{c}\mathrm{DS}\left(N_{\text {local }}=\sqrt{N_{\text {gal }}}\right) \\
P\left(\Delta_{\mathrm{S}}>\Delta_{\text {obs }}\right)(\%)\end{array}$ & $r_{\mathrm{sp}}$ & $r_{\mathrm{cp}}$ & $\begin{array}{c}\mathrm{DS}\left(N_{\text {local }}=10\right) \\
P\left(\Delta_{\mathrm{S}}>\Delta_{\mathrm{obs}}\right)(\%)\end{array}$ & $\begin{array}{c}\operatorname{DS}\left(N_{\text {local }}=\sqrt{N_{\text {gal }}}\right) \\
P\left(\Delta_{\mathrm{s}}>\Delta_{\text {obs }}\right)(\%)\end{array}$ \\
\hline A963 & 1.95 & 1.33 & $0.06 \pm 0.06$ & 0.30 & 0.01 & 0.03 \\
\hline A2261 & 21.20 & 14.32 & $0.1 \pm 0.09$ & 0.63 & 1.17 & 1.36 \\
\hline A1423 & 18.90 & 19.30 & $0.07_{-0.07}^{+0.13}$ & 0.31 & 7.43 & 7.16 \\
\hline A611 & 6.10 & 6.94 & $0.19 \pm 0.11$ & 0.39 & 8.05 & 1.58 \\
\hline RXJ2129 & 35.20 & 20.17 & $0^{+0.15}$ & 0.48 & 13.99 & 14.64 \\
\hline MACSJ1206 & 27.88 & 15.06 & $0.07 \pm 0.04$ & 0.15 & 0.69 & 0.16 \\
\hline CL2130 & 0.20 & 0.22 & $0.06_{-0.06}^{+0.09}$ & 0.81 & 0.20 & 0.17 \\
\hline
\end{tabular}

Notes. Columns under D99 and HK96 are estimations made after removing interlopers using the D99 and HK96 procedures, respectively. Columns 2 and 6 are substructure levels using the DS test when $N_{\text {local }}=10$, and Columns 3 and 7 are the same but for $N_{\text {local }}=\sqrt{N_{\text {gal }}}$. Columns 4 and 5 are relaxation estimations, after cleaning interlopers using the D99 method, using the $r_{\mathrm{sp}}$ and $r_{\mathrm{cp}}$ proxies, respectively.

In most cases, taking $N_{\text {local }}=\sqrt{N_{\text {gal }}}$ gives about the same or lower DS values in both interloper removal methods. This reinforces Bird's (1994) claim that using a non-constant value for the number of nearest neighbors increases the sensitivity of this test to significant structure. Another outcome is that removing interlopers with HK96 gives, in most cases, a larger substructure level than with D99. Since the D99 method, on average, gives a lower substructure level, we use it to clean interlopers before we define accreted and infalling satellite halos.

\subsection{The Contribution of Different Mass Ratios to Cluster Growth}

In this section, we first show the $2 \mathrm{D}$ spatial distribution of the cluster members and infalling galaxies when the galaxies are identified using the D99 method. Then, out of these bound galaxies, we identify accreted and infalling satellite halos and estimate their dynamical properties. Finally, we estimate the differential fraction of the cluster mass accreted and compare it to our simulation-based predictions (see Section 4).

In Figure 2, we show for each cluster its cluster members and infalling galaxies (black and red dots) and their smoothed surface density. We identify halos using the two HFs described in Section 3.4. The white contours are overdensities found by using the overdensity HF (see Section 3.4.1). Red dots are galaxy groups identified using the FoF HF (see Section 3.4.2). White circles mark the virial radius estimated using the caustic mass profile.

In Table 6 , we present the properties of all the $2 \sigma$ regions (including the clusters' cores) identified by the overdensity HF. This includes the galaxy surface density peaks, virial and projected masses, $P\left(\Delta_{\mathrm{s}}>\Delta_{\mathrm{obs}}\right)$, LOS velocities (after removing the cluster mean velocity), mean redshifts, and number of galaxies. Here we use the projected and virial mass estimations and not the caustics ones, since in these identified regions there are low numbers of galaxies, $\lesssim 100$, and the caustic mass estimation is less reliable in such cases (M. Geller 2012, private communication).

We take each identified satellite halo and estimate its mass ratio, $\zeta \equiv M_{\text {small }} / M_{\mathrm{mp}}$, assuming $M_{\mathrm{mp}} \approx M_{\text {cluster }}$ (for more details, see Section 4). For $M_{\text {small }}$ and $M_{\mathrm{mp}}$, we use the uncorrected projected mass estimator, since many of the satellite halos do not have enough galaxies for the correction. Thus, the accreted and infalling satellite halos' masses are $M_{\text {small }}=M_{\text {proj }}$ (taken from Table 6). For consistency, in estimating $\zeta$, we use the same mass estimator to estimate the cluster mass, which is taken to be the mass inside the virial radius, i.e., $M_{\text {cluster }}=M_{\text {proj,vir }}$ (the last column in Table 4). We divide the mass ratio range to a low number of bins, 3, because we have a low number of satellites. For each bin, we estimate the differential fraction of the cluster mass accreted, $F(\zeta)$. To estimate $F(\zeta)$, we calculate for each cluster the sum of the masses of all identified satellite halos in the relevant $\zeta$ range. Then to be consistent with our predictions, 

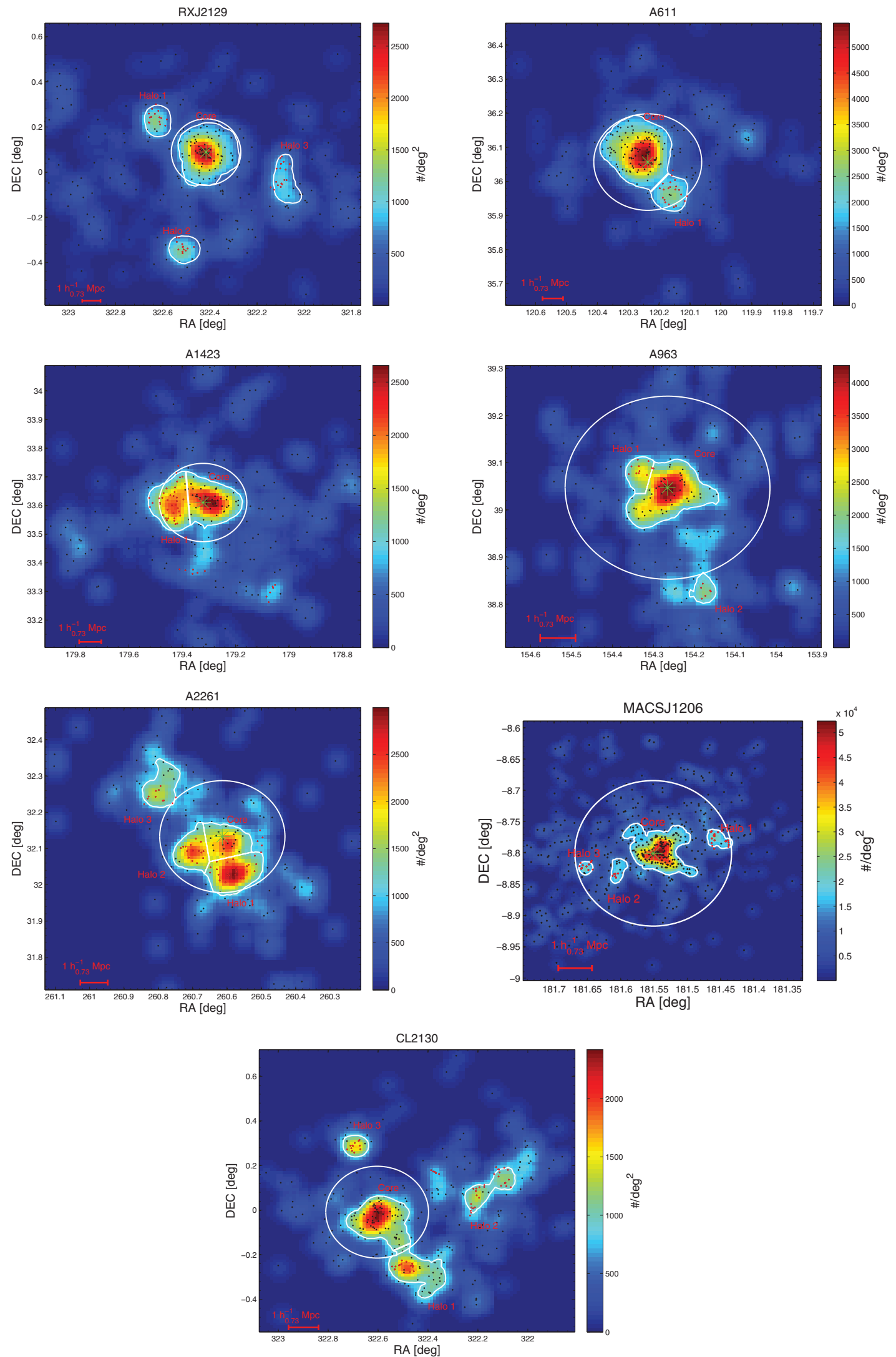

Figure 2. Bound galaxies' surface density and identified halos. Cluster members and infalling galaxies are identified using the D99 method. The white contours are overdensities found by using the overdensity HF (see Section 3.4.1). The galaxies' surface densities are smoothed, and galaxy surface density peaks with $\sim 3 \sigma$ above the average are identified. White contours surround galaxy surface densities corresponding to a significance of $\sim 2 \sigma$ above the average. Red galaxies are groups identified using the FoF HF (see Section 3.4.2) with $b_{\text {frac }}=0.54$ and $N_{\text {gal,min }}=6$. Green asterisks mark the X-ray peaks (when exists). White circles mark the virial radius estimated using the caustic mass profile.

(A color version of this figure is available in the online journal.) 
Information about the $2 \sigma$ Regions Identified by the Overdensity HF

\begin{tabular}{|c|c|c|c|c|c|c|c|c|c|c|c|c|}
\hline Cluster Field & Halo name & $\begin{array}{l}\text { R.A. } \\
\text { (deg) }\end{array}$ & $\begin{array}{l}\text { Decl. } \\
\text { (deg) }\end{array}$ & $\begin{array}{c}M_{\text {vir }} \\
\left(10^{14} h_{0.73}^{-1} M_{\odot}\right)\end{array}$ & $\begin{array}{c}M_{\text {proj }} \\
\left(10^{14} h_{0.73}^{-1} M_{\odot}\right)\end{array}$ & $\begin{array}{c}P\left(\Delta_{\mathrm{s}}>\Delta_{\mathrm{obs}}\right) \\
(\%)\end{array}$ & $r_{\mathrm{sp}}$ & $r_{c p}$ & $\begin{array}{c}v_{\text {los }} \\
\left(\mathrm{km} \mathrm{s}^{-1}\right)\end{array}$ & $z$ & $\begin{array}{c}\sigma^{\mathrm{a}} \\
\left(\mathrm{km} \mathrm{s}^{-1}\right)\end{array}$ & $\overline{N_{\text {gal }}}$ \\
\hline A963 & Core & $154.2563 \pm 0.0117$ & $39.0500 \pm 0.0117$ & $8.93 \pm 1.35$ & $11.17 \pm 1.95$ & 60.22 & $0.32 \pm 0.30$ & 0.44 & $-80 \pm 4$ & 0.2037 & $946 \pm 2$ & 52 \\
\hline A963 & Halo 1 & $154.3264 \pm 0.0117$ & $39.0733 \pm 0.0117$ & $6.63 \pm 2.84$ & $7.23 \pm 1.68$ & 41.79 & $0.57_{-0.57}^{+0.67}$ & 0.35 & $117 \pm 11$ & 0.2045 & $1218 \pm 8$ & 10 \\
\hline A963 & Halo 2 & $154.1785 \pm 0.0117$ & $38.8242 \pm 0.0117$ & $0.26 \pm 0.30$ & $0.25 \pm 0.13$ & 75.18 & $0.84 \pm 0.81$ & 0.60 & $-172 \pm 12$ & 0.2033 & $354 \pm 5$ & 6 \\
\hline A2261 & Core & $260.6006 \pm 0.0139$ & $32.1134 \pm 0.0139$ & $3.21 \pm 0.96$ & $3.57 \pm 0.89$ & 52.19 & $0^{+0.45}$ & 0.34 & $-101 \pm 7$ & 0.2246 & $649 \pm 5$ & 20 \\
\hline A2261 & Halo 1 & $260.5820 \pm 0.0139$ & $32.0298 \pm 0.0139$ & $5.01 \pm 1.33$ & $4.61 \pm 0.96$ & 18.27 & $0.45 \pm 0.40$ & 0.43 & $244 \pm 7$ & 0.2260 & $743 \pm 28$ & 27 \\
\hline A2261 & Halo 2 & $260.7027 \pm 0.0139$ & $32.0948 \pm 0.0139$ & $3.03 \pm 0.99$ & $3.02 \pm 0.93$ & 16.90 & $0.64 \pm 0.49$ & 0.67 & $141 \pm 8$ & 0.2256 & $615 \pm 11$ & 18 \\
\hline A2261 & Halo 3 & $260.8049 \pm 0.0139$ & $32.2527 \pm 0.0139$ & $2.62 \pm 0.64$ & $2.68 \pm 0.58$ & 10.27 & $2.55 \pm 0.49$ & 1.69 & $-355 \pm 9$ & 0.2236 & $562 \pm 13$ & 15 \\
\hline A1423 & Core & $179.3047 \pm 0.0179$ & $33.6144 \pm 0.0179$ & $3.85 \pm 0.78$ & $4.60 \pm 1.05$ & 3.10 & $0.21_{-021}^{+0.37}$ & 0.37 & $104 \pm 9$ & 0.2144 & $590 \pm 45$ & 49 \\
\hline A1423 & Halo 1 & $179.4242 \pm 0.0179$ & $33.6024 \pm 0.0179$ & $4.89 \pm 0.92$ & $5.13 \pm 0.96$ & 10.52 & $0.85 \pm 0.41$ & 0.69 & $-128 \pm 6$ & 0.2135 & $642 \pm 3$ & 38 \\
\hline A611 & Core & $120.2534 \pm 0.0155$ & $36.0763 \pm 0.0155$ & $12.55 \pm 1.48$ & $14.78 \pm 1.70$ & 56.32 & $0.49 \pm 0.29$ & 0.15 & $116 \pm 3$ & 0.2875 & $883 \pm 17$ & 104 \\
\hline A611 & Halo 1 & $120.1500 \pm 0.0155$ & $35.9626 \pm 0.0155$ & $2.61 \pm 0.85$ & $2.55 \pm 0.80$ & 93.55 & $0.96 \pm 0.56$ & 0.72 & $-219 \pm 7$ & 0.2861 & $568 \pm 3$ & 20 \\
\hline RXJ2129 & Core & $322.4170 \pm 0.0220$ & $0.0898 \pm 0.0220$ & $6.86 \pm 1.28$ & $7.95 \pm 1.35$ & 9.86 & $0^{+0.29}$ & 0.02 & $-46 \pm 4$ & 0.2338 & $631 \pm 15$ & 79 \\
\hline RXJ2129 & Halo 1 & $322.0791 \pm 0.0220$ & $-0.0424 \pm 0.0220$ & $2.98 \pm 1.75$ & $3.84 \pm 1.44$ & 37.11 & $0.49_{-0.49}^{+0.52}$ & 0.88 & $-129 \pm 8$ & 0.2335 & $516 \pm 3$ & 20 \\
\hline RXJ2129 & Halo 2 & $322.6227 \pm 0.0220$ & $0.2367 \pm 0.0220$ & $1.78 \pm 0.47$ & $1.68 \pm 0.43$ & 1.12 & $0.82 \pm 0.61$ & 0.62 & $-47 \pm 8$ & 0.2338 & $420 \pm 4$ & 17 \\
\hline RXJ2129 & Halo 3 & $322.5199 \pm 0.0220$ & $-0.3362 \pm 0.0220$ & $1.68 \pm 0.58$ & $1.70 \pm 0.42$ & 23.54 & $0^{+0.61}$ & 0.07 & $586 \pm 9$ & 0.2364 & $484 \pm 2$ & 16 \\
\hline MACSJ1206 & Core & $181.5380 \pm 0.0042$ & $-8.7953 \pm 0.0042$ & $10.74 \pm 1.12$ & $13.29 \pm 1.56$ & 74.87 & $0.34 \pm 0.18$ & 0.24 & $41 \pm 13$ & 0.4402 & $1041 \pm 88$ & 149 \\
\hline MACSJ1206 & Halo 1 & $181.4591 \pm 0.0042$ & $-8.7727 \pm 0.0042$ & $2.45 \pm 0.88$ & $2.50 \pm 0.68$ & 53.46 & $0^{+0.48}$ & 0.91 & $243 \pm 32$ & 0.4412 & $878 \pm 37$ & 18 \\
\hline MACSJ1206 & Halo 2 & $181.6057 \pm 0.0042$ & $-8.8375 \pm 0.0042$ & $3.98 \pm 1.38$ & $7.34 \pm 3.41$ & 79.84 & $0.48_{-0.48}^{+0.51}$ & 1.11 & $119 \pm 43$ & 0.4406 & $1006 \pm 43$ & 13 \\
\hline MACSJ1206 & Halo 3 & $181.6536 \pm 0.0042$ & $-8.8263 \pm 0.0042$ & $3.07 \pm 1.52$ & $3.22 \pm 1.38$ & 79.34 & $1.06 \pm 0.72$ & 0.78 & $-153 \pm 44$ & 0.4393 & $1022 \pm 12$ & 9 \\
\hline CL2130 & Core & $322.6034 \pm 0.0192$ & $-0.0090 \pm 0.0192$ & $4.17 \pm 0.53$ & $4.99 \pm 0.71$ & 1.29 & $0.20_{-020}^{+0.30}$ & 0.68 & $-67 \pm 4$ & 0.1357 & $607 \pm 10$ & 65 \\
\hline CL2130 & Halo 1 & $322.4756 \pm 0.0192$ & $-0.2646 \pm 0.0192$ & $1.73 \pm 0.53$ & $3.53 \pm 1.20$ & 9.61 & $0.33_{-0.33}^{+0.20}$ & 0.60 & $-320 \pm 6$ & 0.1348 & $417 \pm 5$ & 42 \\
\hline CL2130 & Halo 2 & $322.2072 \pm 0.0192$ & $0.0549 \pm 0.0192$ & $2.04 \pm 0.83$ & $3.31 \pm 1.02$ & 0.15 & $0.94 \pm 0.47$ & 1.16 & $718 \pm 7$ & 0.1387 & $528 \pm 6$ & 24 \\
\hline CL2130 & Halo 3 & $322.6801 \pm 0.0192$ & $0.2849 \pm 0.0192$ & $0.33 \pm 0.11$ & $0.40 \pm 0.10$ & 11.49 & $0.90 \pm 0.67$ & 0.44 & $-834 \pm 9$ & 0.1328 & $271 \pm 6$ & 14 \\
\hline
\end{tabular}

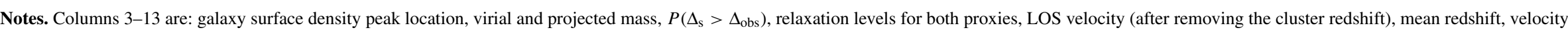
dispersion, and number of galaxies in the $2 \sigma$ regions identified by the overdensity HF.

${ }^{a}$ The velocity dispersion uncertainty here is only due to measurements uncertainties. 

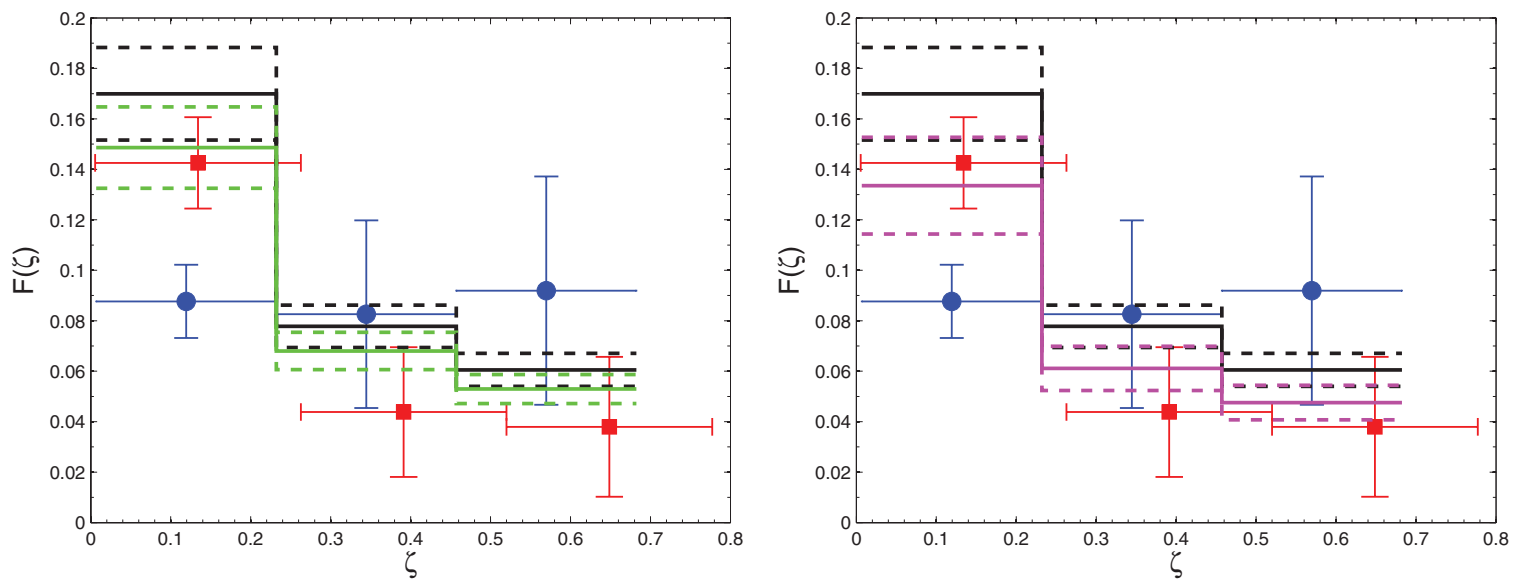

Figure 3. Differential fraction of the cluster mass accreted, $F(\zeta)$, vs. the halo mass ratio, $\zeta$. Blue circles and red squares are for satellite halos identified by the overdensity and FoF HFs, respectively. Left panel: black (green) curves are our simulation-based predictions (for more details, see Section 4) using WMAP7 cosmology and $\alpha=0.15(\alpha=0.133)$. Right panel: black (purple) curves are our simulation-based predictions using WMAP7 (WMAP1) cosmology. Scaling by $\sigma_{8}$, the theoretical expectation using WMAP9 is in between the expectations from WMAP1 and WMAP7 and closer to the latter. In both panels, solid and dashed curves represent the simulation-based predictions' mean and $1 \sigma$ uncertainty, respectively.

(A color version of this figure is available in the online journal.)

we divide it by the cluster mass at the time when all identified matter falls into it, i.e., $z_{1}$ (for more details see Section 4.2), using Equation (13). Finally, we average out $F(\zeta)$ over all the clusters.

For estimating the $F(\zeta)$ uncertainties, we make $10^{2}$ realizations where in each one and for each cluster we randomly take a value from a Gaussian distribution for both the infall or accreted satellite and cluster masses. The Gaussian mean and standard deviation are taken to be the mass estimation and its uncertainty, respectively. For each realization and for each cluster, we estimate $F(\zeta)$ as is explained above. Then, we average out $F(\zeta)$ over all clusters. Finally, we estimate the $F(\zeta)$ uncertainties as the standard deviation of all the realized $F(\zeta)$.

In Figure 3, we show the simulation-based theoretical expectation of $F(\zeta)$ that we derive following the procedure described in Section 4. For $M_{\mathrm{obs}}$, we insert the clusters' $M_{\text {proj, vir }}$ (for consistency with observational based $\zeta$ values). We first estimate the expected $F(\zeta)$ for each cluster by inserting the cluster's $M_{\mathrm{obs}}, z_{\mathrm{c}}$, and $z_{\mathrm{f}}$ values to Equation (19). Then we take the final theoretical expectation of $F(\zeta)$ (solid curves) and its uncertainty (dashed curve) to be the mean and standard deviation, respectively, of the expected $F(\zeta)$ of all clusters.

In the left panel, we present the theoretical expectations using the two different $\alpha$ values mentioned in Section 4, 0.15 and 0.133 (black and green curves, respectively). As is mentioned in Section 4.1, Angulo et al. (2012) scaled the Millennium simulations, which are based on the values found by analyzing $W M A P 1$, to the ones based on the values found by analyzing WMAP7. In the right panel, we present the theoretical expectations using two different cosmologies WMAP7 and $W M A P 1$ (black and purple curves, respectively). Scaling by $\sigma_{8}$, the theoretical expectations using WMAP9 are in between the expectations from WMAP1 and WMAP7 and closer to the latter. On top of the predicted $F(\zeta)$, we plot our derived values using the two different HFs, i.e., overdensity (blue circles) and FoF (red squares). The theoretical expectation $F(\zeta)$ bins are the same as the ones of the estimated $F(\zeta)$ using the overdensity HF.

In Figure 4, we plot the infalling and accreted satellite mass histogram, where the satellite halos are identified using the two different HFs, i.e., overdensity (blue circles) and FoF (red

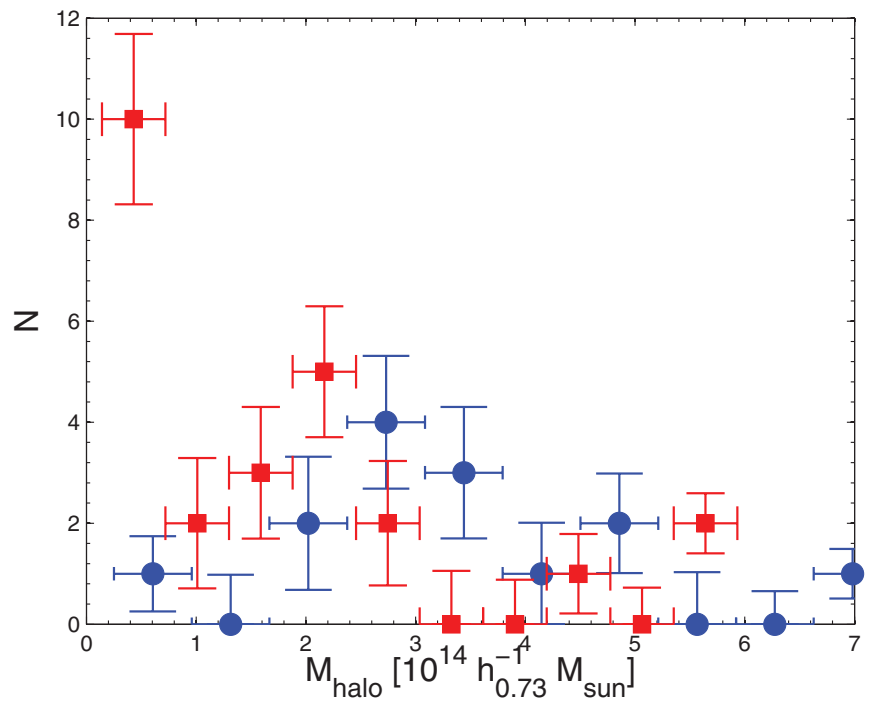

Figure 4. Histogram of the infall and accreted satellites' masses. Blue circles and red squares are for halos identified by the overdensity and FoF HFs, respectively. (A color version of this figure is available in the online journal.)

squares). In both halo definitions, at masses lower than about $2 \times 10^{14} h_{0.73}^{-1} M_{\odot}$, the efficiency of detecting halos decreases. Dividing this number by the averaged cluster mass, which again is taken to be $M_{\text {proj,vir }}$, we get $\zeta \sim 0.14$. Thus, at the $\zeta$ bin with the lowest value, the number of identified halos is underestimated.

The $\zeta$ bin with the highest value may be underestimated as well, if mergers with high $\zeta$ are missed. These mergers may be missed for two reasons. First, all the clusters in our sample (except for CL2130) were chosen to be quite X-ray relaxed (P12). Therefore, this sample is biased against large $\zeta$ values. Second, mergers with large $\zeta$ are more rare. Taking Equation (16) (with our clusters' masses and redshifts and integrating over the $z_{\mathrm{f}} \leqslant z \leqslant z_{\mathrm{c}}$ redshift range), we expect the averaged (over all clusters) number of mergers per cluster to be $\approx 0.24 \pm 0.05$ and $\approx 0.21 \pm 0.04$ for $N_{\mathrm{m}}(\zeta \gtrsim 0.7, \alpha=0.15)$ and $N_{\mathrm{m}}(\zeta \gtrsim 0.7, \alpha=0.133)$, respectively. This gives a total 

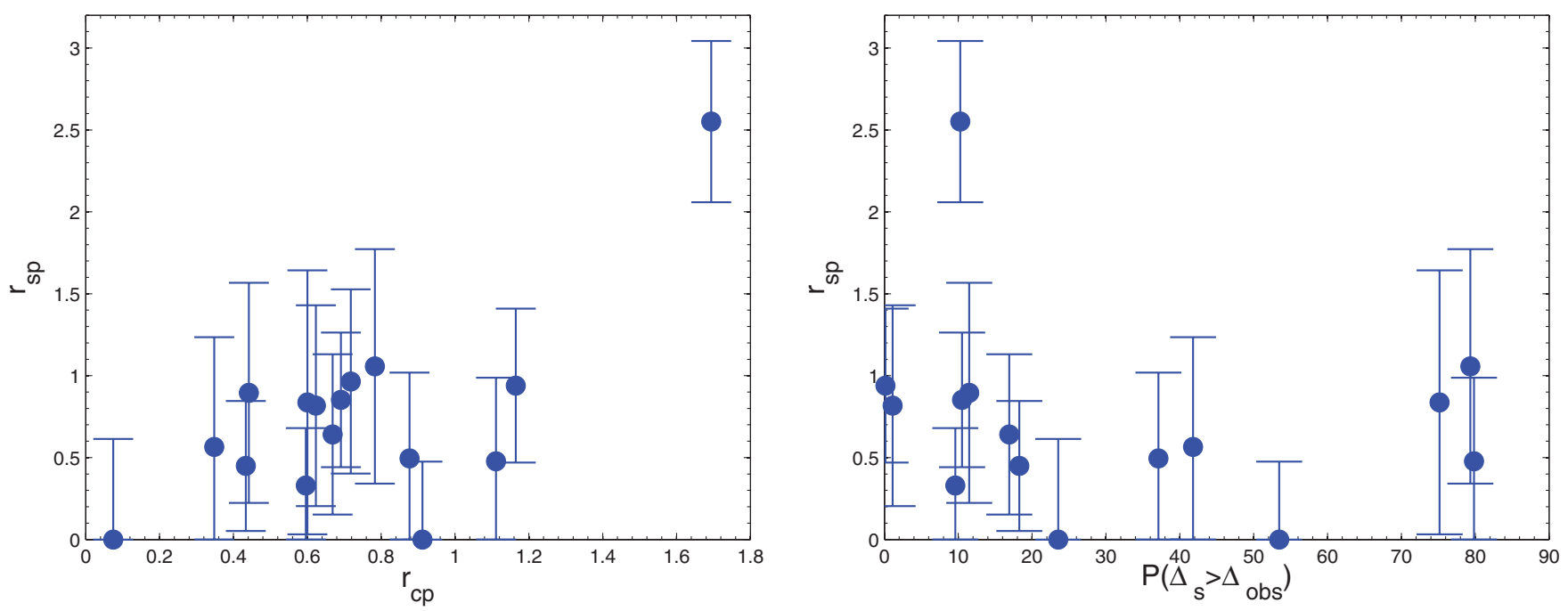

Figure 5. Left panel: estimated relaxation levels using the two proxies discussed in Section 3.5.2, $r_{\mathrm{sp}}$ and $r_{\mathrm{cp}}$, for the infalling and accreted satellite halos. Right panel: estimated substructure levels (using the DS test) vs. the relaxation levels (using the $r_{\text {sp }}$ proxy) for the same halos.

(A color version of this figure is available in the online journal.)

number of $\sim 1$ merger for our seven-cluster sample. Thus, these high $\zeta$ mergers may not be seen due to our sample size.

\subsection{Substructure-Relaxation Connection}

In this section, we test if the correlation between the substructure and relaxation levels of the infalling and accreted satellite halos is strong. In Figure 5 (left panel), we show the measured relaxation levels using the two proxies described in Section 3.5.2, $r_{\mathrm{sp}}$ and $r_{\mathrm{cp}}$. In Figure 5 (right panel), we show the relaxation levels using the $r_{\mathrm{sp}}$ proxy versus substructure levels using the DS test. The substructure levels do not correlate strongly with our estimated dynamical relaxation levels (using the $r_{\text {sp }}$ proxy), while the correlation between the two relaxation proxies is stronger.

\section{DISCUSSION}

In this paper, we estimate the mass and dynamical properties of seven clusters and of the halos falling (and accreted) into them. Then we estimate the contribution of different mass ratios to cluster growth and compare these estimations to our expectations based on simulations.

Before estimating the clusters' dynamical properties, it is important to remove interlopers from them. We test two interloper removal methods, HK96 and D99. We use the fact that the DS test is sensitive to outliers in order to indicate which interloper removal method is most effective. Removing interlopers with HK96 gives a larger substructure level than with D99 (see Table 5). Therefore, the D99 method is better in dealing with the presence of structures that are along the LOS and have a small projected distance to the cluster center. Thus, we use the D99 method to clean interlopers.

Our analysis is heavily based on estimating the masses of clusters and accreted and infalling halos. Thus, we examine four possible mass profile biases. Namely, we check the effect of the interloper removal method, the mass estimator used, the number of identified galaxies, and uneven sampling of the sky's plane. The HK96 method yields wider caustics and, therefore, a higher estimated mass (see Appendix C). The masses estimated, at large radii (i.e., about the virial radius), using the HK96 method were $\sim 50 \%-100 \%$ higher than those estimated using
D99 (when the higher values are for clusters with many massive infalling satellites, e.g., RXJ2129 and CL2130), although, in about $2 \sigma$ confidence level, the two methods yield virial masses which are in agreement. This uncertainty is even greater than the one due to the projection effect, which, for the caustic mass, is about $30 \%$ (for about 100 cluster members within the virial radius; Gifford et al. 2013; S11; Saro et al. 2013; Gifford \& Miller 2013).

Overall, we find a $1 \sigma$ agreement between the caustic, virial, and projected mass profiles. Although the projected mass profile is in $\sim 1 \sigma$ agreement with the caustic mass profile, it is usually higher. Therefore, the derived $r_{200}, r_{\mathrm{vir}}, M_{200}$, and $M_{\mathrm{vir}}$ values for most clusters are higher when using the projected mass profile than the caustics one (see Table 4). This is in agreement with Rines \& Diaferio (2006), who also found that at large radii the projected mass profile tends to be higher than the caustic one. At large radii, the caustic mass uncertainty is larger than the projected (and virial) mass uncertainty (see Figure 1 and Table 4) because it also takes into consideration uncertainty due to the projection effect (though not with exactly $1 \sigma$ confidence level; see the notes in Section 3.3.2 and Appendix B.2.3 for more details).

As expected, when the number of galaxies is smaller, the statistic uncertainty is larger (see Appendix C). Estimating the virial mass profile, taking about $15 \%$ (when these $15 \%$ are 41 and 63 galaxies for A963 and RXJ2129, respectively) and $75 \%$ of the clusters' spectroscopically identified galaxies gave $30 \%-50 \%$ and $5 \%-10 \%$ scatter over the mean, respectively. Uneven sampling of the sky's plane does not insert a large uncertainty (see Appendix C). We test it by excluding a sector and estimating the mass profile. Generally, excluding a sector increases both the statistical and systematic (downward) uncertainties. However, even when excluding a sector with a large opening angle, $\pi / 2$, at the virial radius, the mass is systematically lower by $\lesssim 7.5 \%$, and the statistical uncertainty is $\lesssim 7.5 \%$. If the obscuration covers only part of the sector, the effect will be smaller, of course. The closer the obscured part is to the cluster center, the larger the bias because there are more galaxies at the center.

Since among the two interloper removal methods we test we take the one that removes interlopers more efficiently, the three 
dynamical mass profiles we test are in $1 \sigma$ agreement, and the uncertainty due to uneven sampling on the plane of the sky is not substantial, we conclude that these three mass biases are not substantial in our case. However, a very low number of galaxies increases substantially the statistical uncertainty. Indeed, the uncertainties in the halo mass estimations (see Table 6) are larger than the ones of the clusters (see the last column in Table 4).

We find good agreement between the dynamical mass profiles and the X-ray derived ones at their overlapping radii range, except for A2261, where at small radii, i.e., $r<418 h_{0.73}^{-1} \mathrm{kpc}$, the X-ray profile is $\sim 2-3 \sigma$ above the dynamical mass profile. At these radii, the dynamical mass profile is estimated using spectroscopic data of less than nine galaxies. In addition, in this cluster, the separation between the X-ray and the galaxy surface number density peaks is the largest. The X-ray and dynamical mass profiles are combined together and give a mass profile range from $\sim 10 h_{0.73}^{-1} \mathrm{kpc}$ to beyond the virial radius.

Biviano et al. (submitted) analyzed MACSJ1206 using the same spectroscopic data. They used different procedures, including the use of two other techniques to remove interlopers, and added photometric data in five bands which were derived from Subaru Suprime-Cam, to derive the cluster mass. Still, their cluster mass estimation, $M_{200}=1.4 \pm 0.2 h_{0.7}^{-1} M_{\odot}$, is in excellent agreement with our results. Umetsu et al. (2012) used a combined WL distortion, magnification, and SL analysis of wide-field 5-band Subaru imaging and 16-band HST observations and found that for MACSJ1206 $M_{\mathrm{vir}}=(1.1 \pm 0.2 \pm 0.1) \times$ $10^{15} h^{-1} M_{\odot}$ (at the virial overdensity of 132 ), corresponding to $M_{200}=(0.98 \pm 0.19 \pm 0.10) \times 10^{15} h^{-1} M_{\odot}$. This is in excellent agreement with our (and Biviano et al. (submitted)) results. Coe et al. (2012), who used strong and weak lensing (hereafter SL and WL, respectively), estimated that $M_{\mathrm{vir}}=(2.2 \pm 0.2) \times$ $10^{15} h_{0.7}^{-1} M_{\odot}$ for A2261 when assuming a spherical halo. This value is higher than our dynamically based mass estimations (see Table 4). Okabe et al. (2010), who used high-quality Subaru/Suprime-Cam imaging data to conduct a detailed WL study, found that $M_{\mathrm{vir}}$ is $6.96_{-1.59}^{+2.17}, 6.65_{-1.42}^{+1.75}$, and $6.71_{-1.96}^{+2.73} \times$ $10^{14} h^{-1} M_{\odot}$ for A963, A611, and RXJ2129, respectively. The values they estimated for all clusters are in agreement with our results. Rodríguez-Gonzálvez et al. (2012) estimated the mass of A611 and A1423 using Sunyaev-Zeldovich (hereafter SZ) measurements and found $M_{200}=4_{-0.8}^{+0.7} \times 10^{14} h^{-1} M_{\odot}$ and $M_{200}=(2.2 \pm 0.8) \times 10^{14} h^{-1} M_{\odot}$, respectively. Both are in $\sim 1 \sigma$ agreement with our results. Newman et al. (2013b) used SL and WL and found that $M_{200}$ is $4.07_{-1.19}^{+1.17} \times 10^{14} h_{0.7}^{-1} M_{\odot}$ and $3.63_{-0.81}^{+1.27} \times 10^{14} h_{0.7}^{-1} M_{\odot}$ for A963 and A611, respectively. All these estimations are also in agreement with ours. Overall, there is general good agreement between our dynamically based mass estimations and the ones derived by lensing and SZ. In one case (A2261), the mass estimation based on lensing is higher than our dynamical one. Our results are in general agreement with Geller et al. (2013), who compared the mass profiles based on the caustic technique with WL measurements taken from the literature for 19 clusters. They found that at $3 r_{200}$ the WL overestimates the caustic profile by about $20 \%-30 \%$, probably due to the impact of superposed large-scale structures. However, at $r_{\mathrm{vir}}$, they found the WL and caustic mass profiles in very good agreement.

Turning to halo accretion, accreted and soon to be accreted halos are identified by first using the D99 methodology to identify cluster members and infalling galaxies. Then we define halos using two different HFs, i.e., overdensities of the smoothed galaxy surface density (for more details, see Section 3.4.1) and FoF (for more details, see Section 3.4.2). The use of two different HFs increases the reliability of our results and shows differences arising from the halo identification scheme. The number of identified halos and their sizes depend on the HF (see Figure 2) and the values inserted into their free parameters. Generally, here the FoF HF tends to break halos, which are identified by the overdensity HF, into a few smaller halos. In addition, the FoF HF identifies more low-mass halos with a filament-like appearance (see the fields of A1423 and CL2130 in Figure 2). As a result of these two differences between the two HFs, the estimated $F(0.2 \lesssim \zeta \lesssim 0.7)$ and $F(0.01 \lesssim \zeta \lesssim 0.2)$ are higher and lower when using the overdensity HF. Nevertheless, a general agreement between the HFs is found in both the detection of halos (see Figures 2 and 4 ) and the estimated $F(\zeta)$ values at the $0.2 \lesssim \zeta \lesssim 0.7$ range

In the $0.2 \lesssim \zeta \lesssim 0.7$ range, our observationally based estimation for the $F(\zeta)$ profile is in $\sim 1 \sigma$ agreement with our theoretically predicted one. At low mass ratios, $\zeta \lesssim 0.2$, the estimated $F(\zeta)$ using the overdensity HF is underestimated since the detection efficiency decreases at low masses, $\sim 2 \times$ $10^{14} h_{0.73}^{-1} M_{\odot}$. The estimated $F(\zeta)$ using the FoF HF is not overestimated but only because generally here the FoF HF tends to break halos, which are identified by the overdensity HF, into a few smaller halos, and it identifies more low-mass halos with a filament-like appearance. As a result, too many low-mass halos were identified (see Figure 4). At large mass ratios, $\zeta \gtrsim 0.7$, we do not detect halos probably because all the clusters in this sample (except for CL2130) were chosen to be quite X-ray relaxed (P12). Therefore, this sample is biased against large $\zeta$ values. Another explanation is that these mergers are rare and may be missed due to our sample size. In addition, in cases where a satellite is partly or fully within the cluster, our cluster mass estimation includes part (or all) of the satellite's mass. Therefore, in these cases, $\zeta$ is underestimated. Thus, at large $\zeta$ values, the estimated $F(\zeta)$ is also underestimated.

Using the overdensity HF, we estimate these halos' centers, redshifts, LOS velocities, masses, relaxation states, and substructure levels. Except for the four mass biases we mention above, the mass estimators we use to measure the identified satellites are based on the assumption that the system is in dynamical equilibrium. The mass estimation of unrelaxed halos due to LOS mergers can be overestimated (Takizawa et al. 2010). As we see in Figure 5 (left panel), the halos' relaxation spectrum is quite wide and some of them may not be relaxed, though our displacement relaxation proxies are more sensitive when the merger axis is in the plane of the sky. Therefore, some of the halo masses may be overestimated. Regarding the substructure levels, the mass merger ratio limitation, for which there is a marginal detection, depends on the sample size (P96). Because in these halos there is a low number of spectroscopically identified galaxies, these substructure level estimations are sensitive for a mass merger ratio limitation of about 1/2 (P96). This narrow mass merger ratio range may explain why there is no significant correlation between the substructure and our estimated dynamical relaxation levels.

\section{SUMMARY}

For the first time, we test a key outcome in the $\Lambda \mathrm{CDM}$ cosmological model: the contributions of mergers with different mass ratios to cluster-sized halo growth. At the $0.2 \lesssim \zeta \lesssim 0.7$ range, we find $\mathrm{a} \sim 1 \sigma$ agreement with the cosmological model. 

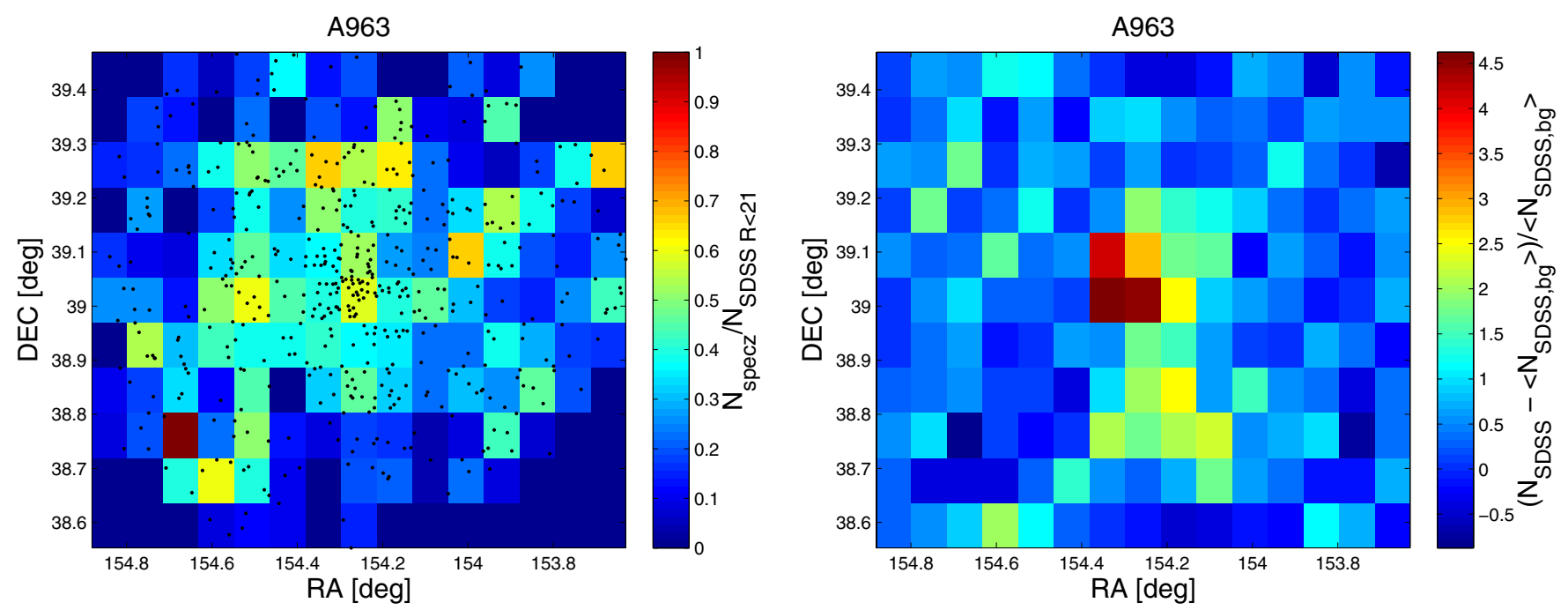

Figure 6. A963 target selection (as described in Section 2.1) plane of the sky completeness. Left panel: the plane of the sky is divided into $5 \times 5$ arcmin regions. Then we calculate in each region the ratio between Hectospec (spectroscopic) sources and SDSS photometric sources which are above the Hectospec limiting $R$ magnitude, i.e., $\sim 21$. Black dots indicate the Hectospec spectroscopically identified galaxies. Right panel: testing real plane of the sky differences in the galaxies' distribution by plotting $\left(N_{\text {SDSS }}-N_{\text {SDSS,bg }}\right) / N_{\text {SDSS, bg }}$, where $N_{\text {SDSS, bg is }}$ the $N_{\text {SDSS }}$ background, which is estimated as the mean of the four $5 \times 5$ arcmin corner regions.

(A color version of this figure is available in the online journal.)

Our other main conclusions are the following.

1. There is good agreement between the caustic, virial, and projected mass profiles.

2. An agreement is found between dynamical and X-ray mass estimations. Our mass estimations at $r_{200}$ and $r_{\mathrm{vir}}$ are also in agreement with most WL and SZ estimations found in the literature.

3. Different interloper removal methods affect the substructure level estimation dramatically, or in other words, the DS test is very sensitive to the method used for removing interlopers.

4. The D99 interloper removal method is more adequate to deal with the presence of close and LOS substructure than HK96.

5. Mass uncertainties can be substantial due to the different cluster members' identification methodology $(\sim 50 \%-100 \%$ at the virial radius, although, in about $2 \sigma$ confidence level, the two methodologies yield virial masses which are in agreement) and the very low number of galaxies $(30 \%-50 \%$ and $5 \%-10 \%$ when taking $15 \%$ and $75 \%$ of the clusters' spectroscopically identified galaxies, respectively). The uncertainty is less substantial in the case of uneven sampling of the sky's plane (when excluding a sector with a large opening angle, $\pi / 2$, at the virial radius, the mass is systematically lower by $\lesssim 7.5 \%$ and the systematic uncertainty is $\lesssim 7.5 \%$ ). All the dynamical mass estimators we used were in agreement within $1 \sigma$, but in most cases the projected mass profiles were higher than the caustic mass profiles.

We thank Margaret J. Geller, Kenneth Rines, Michael Kurtz, and Antonaldo Diaferio for providing their redshift data for A611 and CL2130 and for providing the redshift data for A963, A2261, A1423, and RXJ2129 in advance of publication. We also thank them for many helpful discussions. In addition, we acknowledge very useful discussions with Ana Laura Serra, Dan Gifford, Mark Neyrinck, Yuval Birnboim, Eyal Neistein, and Maxim Markevitch. We thank the anonymous referee for useful comments. D.L. thanks Eran Ofek for his publicly avail- able Matlab scripts. This research is supported in part by NASA grant HST-GO-12065.01-A. M.M. acknowledges support from PRIN INAF 2009 and ASI (agreement Euclid phase B2/C). A.Z. is supported by contract research "Internationale Spitzenforschung II/2-6" of the Baden Württemberg Stiftung.

\section{APPENDIX A}

\section{SURVEY COMPLETENESS}

Target selection (as described in Section 2.1) plane of the sky completeness is checked by comparing those targets to more uniformly selected sources. Hectospec and VIMOS sources are compared to SDSS and Canada France Hawaii Telescope (CFHT) sources, respectively. In Figures 6-10, for each of the clusters observed using Hectospec we divide its plane of the sky into $5 \times 5$ arcmin regions. We then calculate in each region the ratio between the Hectospec (spectroscopic) sources, $N_{\text {specz }}$, and the SDSS photometric galaxies, which are above the Hectospec limiting $R$ magnitude, i.e., $\sim 21, N_{\text {SDSs }}$. This procedure allows us to see if there are regions with large coherent patterns of higher than average incompleteness, which can happen due to bright stars, for example. Regions may look incoherent not due to incompleteness but rather due to real plane of the sky differences in the galaxies' distribution. Therefore, in order to check for the latter case, we also plotted the ratio $\left(N_{\mathrm{SDSS}}-N_{\mathrm{SDSS}, \mathrm{bg}}\right) / N_{\mathrm{SDSS}, \mathrm{bg}}$, where $N_{\text {SDSS, bg }}$ is the $N_{\text {SDSS }}$ background, which is estimated as the mean of the four $5 \times 5$ arcmin corner regions.

In Figure 11, we divide MACSJ1206 plane of the sky into $3 \times 3$ arcmin regions. Then we calculate $N_{\text {specz }} / N_{\text {CFHT }}$ and $\left(N_{\mathrm{CFHT}}-N_{\mathrm{CFHT}, \mathrm{bg}}\right) / N_{\mathrm{CFHT}, \mathrm{bg}}$ for sources with $R \leqslant 24.5$.

Note that in Appendix $C$ we estimate the uncertainty in the virial mass profile due to plane of the sky incompleteness sampling.

\section{APPENDIX B}

\section{TECHNIQUES FOR REMOVAL OF NON-CLUSTER GALAXIES}

In the following two subsections, we briefly describe the two methods we use to remove interlopers. 

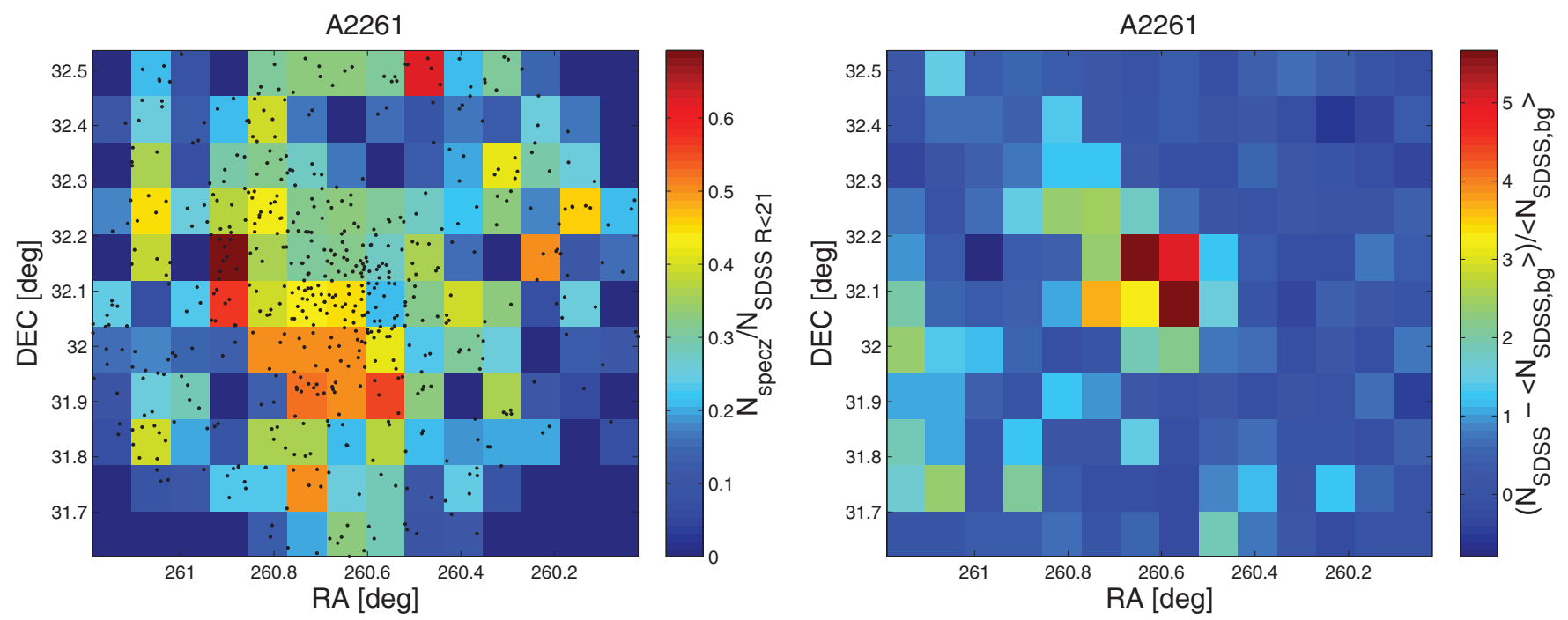

Figure 7. Same as Figure 6 but for A2261.

(A color version of this figure is available in the online journal.)
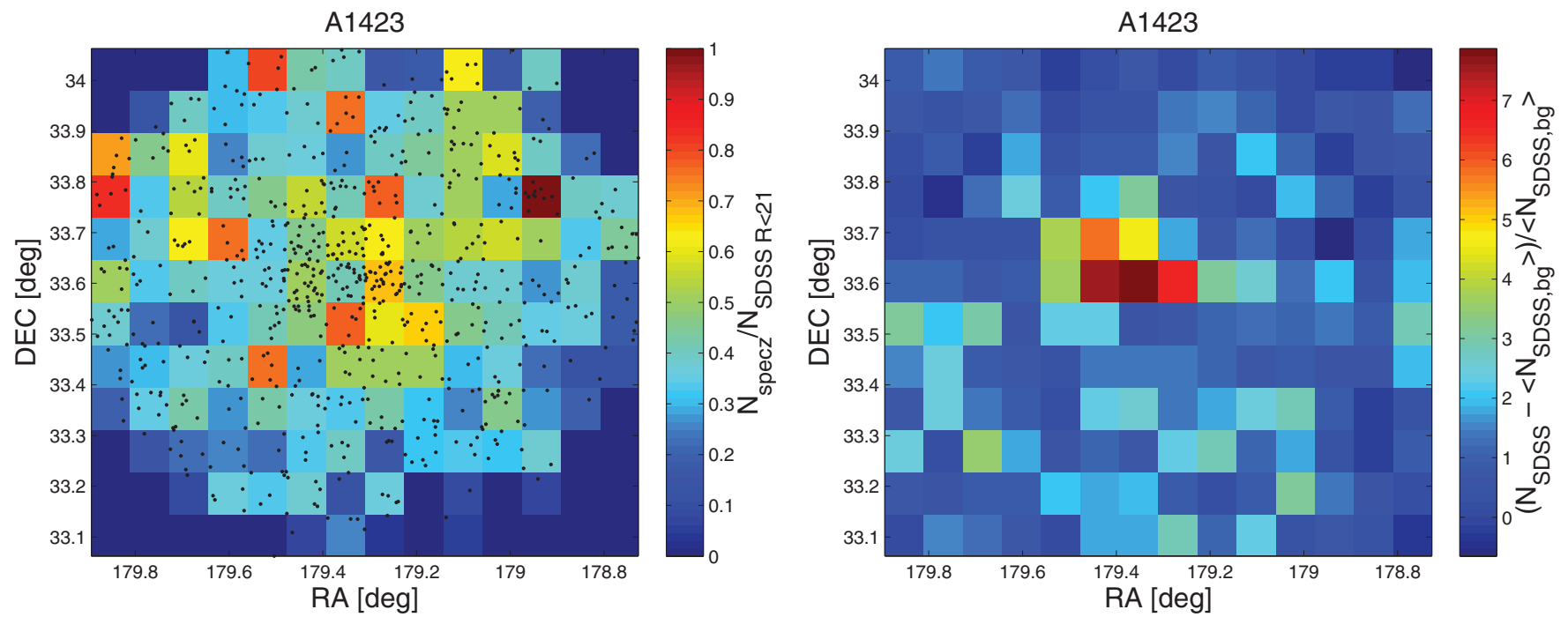

Figure 8. Same as Figure 6 but for A1423.

(A color version of this figure is available in the online journal.)
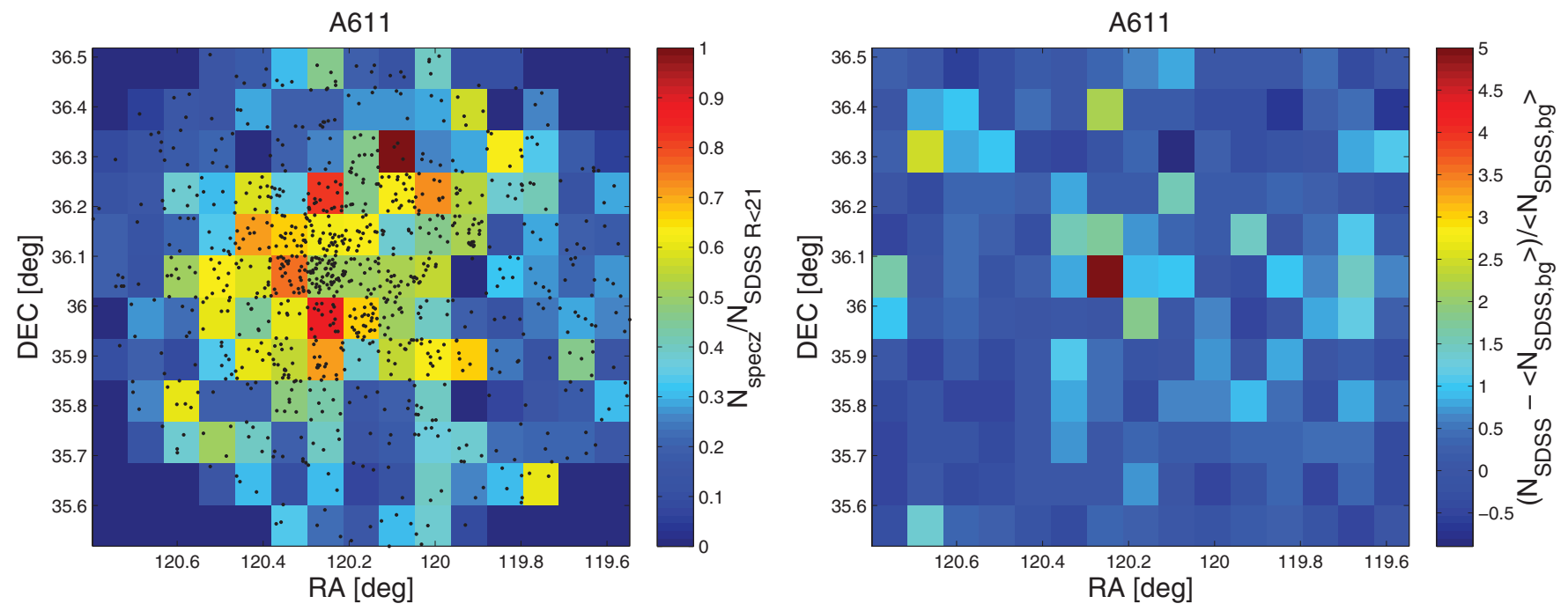

Figure 9. Same as Figure 6 but for A611.

(A color version of this figure is available in the online journal.) 

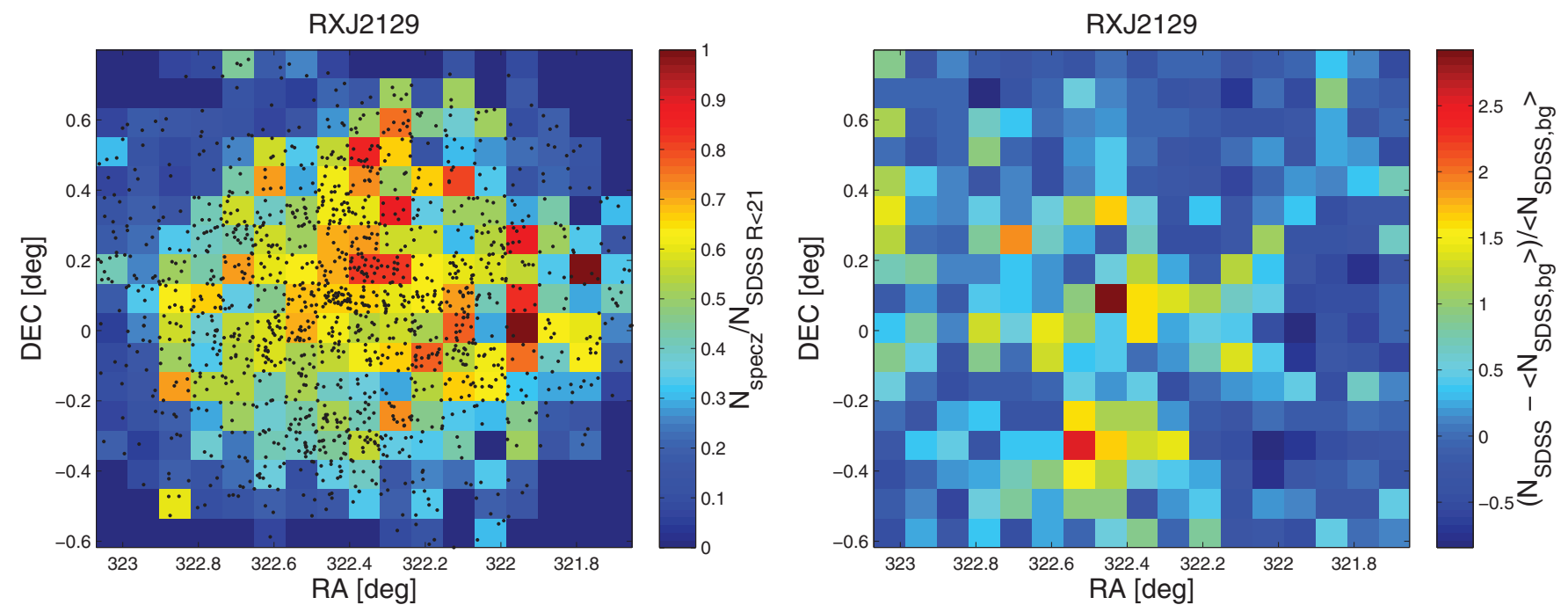

Figure 10. Same as Figure 6 but for RXJ2129.

(A color version of this figure is available in the online journal.)
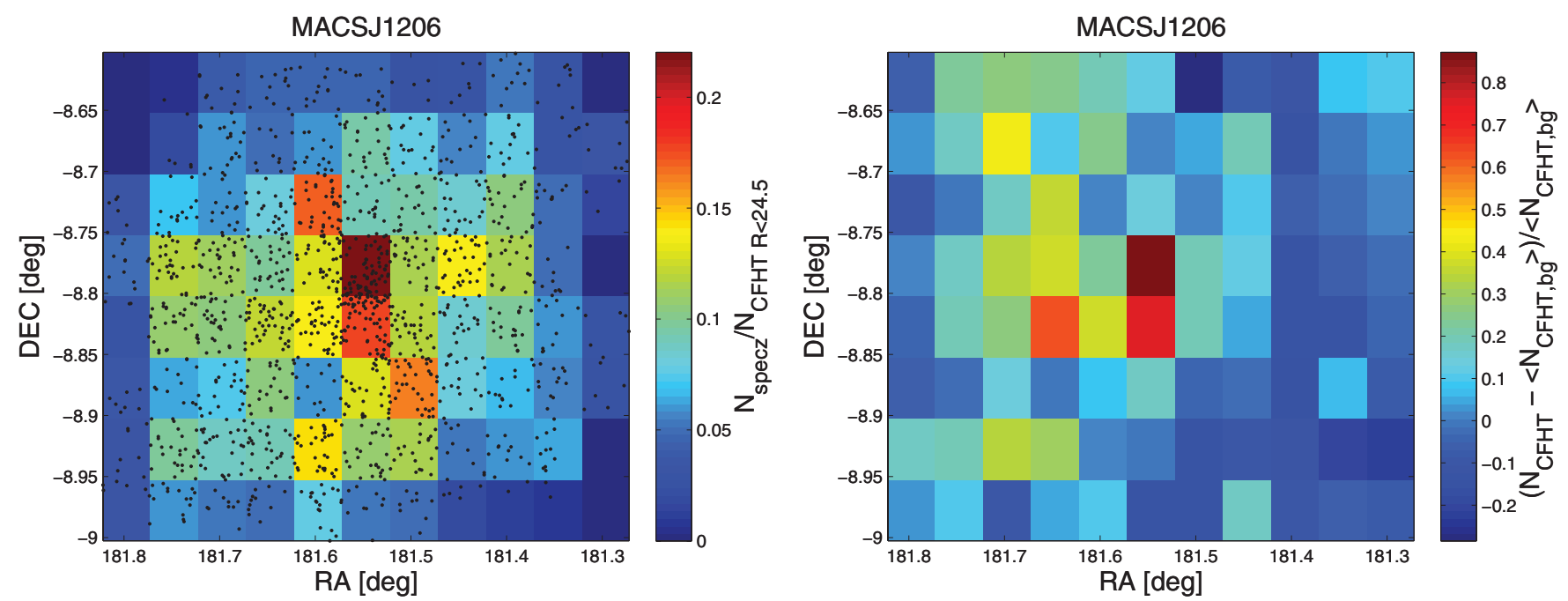

Figure 11. MACSJ1206 target selection (as described in Section 2.1) plane of the sky completeness. Left panel: the plane of the sky is divided into $3 \times 3$ arcmin regions. Then we calculate in each region the ratio between VIMOS (spectroscopic) sources and CFHT photometric sources with $R \leqslant 24.5$. Black dots indicate the VIMOS spectroscopically identified galaxies. Right panel: testing real plane of the sky differences in the galaxies' distribution by plotting $\left(N_{\mathrm{CFHT}}-N_{\mathrm{CFHT}}\right.$,bg $) / N_{\mathrm{CFHT}}$,bg, where $N_{\mathrm{CFHT}}$,bg is the $N_{\mathrm{CFHT}}$ background, which is estimated as the mean of the four $3 \times 3$ arcmin corner regions.

(A color version of this figure is available in the online journal.)

\section{B.1. den Hartog \& Katgert Interloper Removal Method}

The HK96 procedure is an iterative method, which uses the estimated mass profile (and assuming spherical symmetry) to infer the maximum LOS velocity profile that a cluster member galaxy can have. We make an initial cut in the velocities as described in Section 3.1. Then in each iteration a mass profile is estimated and the maximum LOS velocity profile is inferred. Two options for dynamically based mass profiles are the virial and projected (see Section 3.3.1). Here we choose to use the projected mass profile (choosing the virial mass profile yields similar results). Galaxies with LOS velocities exceeding the maximum LOS velocity at their projected radius are considered to be interlopers and are removed. Then we estimate a new mass profile and so on. The method converges in 1 to 10 iterations.

The maximum LOS velocity profile is calculated as follows. Since the velocity anisotropy is not known, galaxies are considered to have circular (tangential) or infalling (radial) orbits. The circular velocity is $v_{\text {cir }}(r)=\sqrt{G M(r) / r}$, and if all orbits are circular with this velocity, the cluster is in virial equilib- rium, since $T_{\text {cir }}=(1 / 2) \int \rho(r) v_{\text {cir }}^{2}(r) d r=-(1 / 2) U$. For bound galaxies, the upper limit for the infall velocity is $T_{\text {infall }}=|U|$, so $v_{\text {infall }}(r)=\sqrt{2} v_{\text {cir }}(r)$.

We ignore the presence of the virialized central region and assume that each galaxy is either on a radial orbit toward the center of the cluster or on a purely circular orbit. Under the first assumption, we calculate the upper limit to the LOS velocities, $V_{\max }(R)$, at projected distance $R$ as the maximum value of the LOS component of the infall velocity for all positions $r$ on the line of sight within the turnaround radius, i.e., $v_{\text {infall }}\left(r_{\max }\right) \cos (\theta)$. Under the second assumption, one uses the maximum LOS component of the circular velocity, i.e., $v_{\text {cir }}\left(r_{\max }\right) \sin (\theta)$. Combining both assumptions yields

$$
\begin{aligned}
V_{\max }(R)= & \max \left\{v_{\text {infall }}\left[r_{\max }(R)\right]\right. \\
& \left.\times \cos [\theta(R)], v_{\text {cir }}\left[r_{\max }(R)\right] \sin [\theta(R)]\right\},
\end{aligned}
$$

where $\theta(R)$ is the angle between the radial vector $r$ and the LOS at $R$, and $r_{\max }(R)$ is the position (along the LOS at $R$ ) where the maximum occurs. 


\section{B.2. Caustics Interloper Removal Method}

The caustic method has a few steps. First, we identify the cluster member candidates using a binary tree. These candidates determine the cluster's velocity dispersion and size. Then these two parameters together with all the galaxies after the velocity cut (see Section 3.1) are used to determine the velocity space density threshold $\kappa$, which determines the caustics and the final cluster members. Below we describe each of these steps in more details.

\section{B.2.1. Binary Tree}

A binary tree is a method to estimate the similarity between the different galaxies. In each step, the two galaxies with the highest similarity are combined into one group. The process continues until all galaxies are joined to one group, which is called the root of the binary tree. Here we use this method to find cluster member candidates, which are needed for the D99 procedure.

As Serna \& Gerbal (1996) suggested, we use the galaxy pairwise binding energy as a measure of similarity:

$$
E_{i, j}=-G \frac{m_{i} m_{j}}{\left|\mathbf{r}_{i}-\mathbf{r}_{j}\right|}+\frac{1}{2} \frac{m_{i} m_{j}}{m_{i}+m_{j}}\left(\mathbf{v}_{i}-\mathbf{v}_{j}\right)^{2},
$$

where $G$ is the gravitational constant and $m_{i}, m_{j}, \mathbf{r}_{i}, \mathbf{r}_{j}, \mathbf{v}_{i}, \mathbf{v}_{j}$ are the masses, positions, and velocities of the two galaxies. To calculate Equation (B2), one needs the full velocity space information (6D), though in our case only three coordinates are available. However, Serna \& Gerbal (1996) found that the projection instability between $6 \mathrm{D}$ and $3 \mathrm{D}$ is quite low, which means that the "observed" structures are reasonably similar to the "intrinsic" 6D ones. Therefore, we used Equation (B2) as our similarity measurement when the $2 \mathrm{D}$ spatial position, $\mathbf{R}$, and the velocities are the LOS ones, $v$, so

$$
E_{i, j}=-G \frac{m_{i} m_{j}}{\left|\mathbf{R}_{i}-\mathbf{R}_{j}\right|}+\frac{1}{2} \frac{m_{i} m_{j}}{m_{i}+m_{j}}\left(v_{i}-v_{j}\right)^{2} .
$$

To build the binary tree, we proceed as follows: (1) Each galaxy is a group $G_{\nu}$. (2) We compute the similarity between each two groups $G_{\mu}, G_{\nu}$ with the single linkage method: $E_{\mu \nu}=\min \left\{E_{\mathrm{ij}}\right\}$, where $E_{\mathrm{ij}}$ is the similarity between the member $i \in G_{\mu}$ and the member $j \in G_{\nu}$. (3) We replace the two groups with the largest similarity (smallest binding energy $E_{\mu \nu}$ ) with a group $G_{\mathrm{k}}$. The number of independent groups is decreased by one. (4) The procedure is repeated from (2) until we are left with only one independent group.

\section{B.2.2. Identifying the Cluster Member Candidates}

After building the binary tree (as is described above and taking $m_{i}=m_{j}=10^{12} h_{0.73}^{-1} M_{\odot}$ to be consistent with S11), we need to identify the cluster member candidates. D99 suggested doing so by first identifying the main branch of the binary tree. The binary tree starts at the root when there is only one group, which is actually the end product of the binary tree procedure. We walk through the tree along the main branch. At each step, or node, the main branch splits into two branches. The branch with the larger number of galaxies is the continuation of the main branch. The velocity dispersion, $\sigma_{x}$, of the galaxies hanging from a given node, $x$, shows a characteristic behavior when walking toward the leaves along the main branch. Initially (at the root of the tree where the galaxy pairwise binding energy is the highest) it decreases rapidly, then it reaches a plateau and again drops rapidly toward the end of the walk. D99 claimed that the plateau, $\sigma_{p l}$, is a clear indication of the presence of the nearly isothermal cluster: at the beginning of the walk, $\sigma_{x}$ is large because we include galaxies outside the main halo; at the end of the walk $\sigma_{x}$ is low since we consider the very central galaxies of a given subclump.

The two nodes $x_{1}$ and $x_{2}$ that limit the $\sigma_{p l}$ plateau are good candidates for the substructure and cluster identification, respectively. Locating $x_{1}$ and $x_{2}$ and estimating sigma plateau, $\sigma_{p l}$, is not a trivial procedure. Their values depend on the estimation procedure and on the definition of similarity used to build the binary tree (which may change a little the $\sigma_{x}-x$ diagram). D99, who applied the method to dissipationless cosmological $N$-body simulations where galaxies form and evolve according to semi-analytic modeling, used a hyperbolic function to fit the plateau and to choose the substructure and cluster thresholds as the inflection points of that fit, while S11, who applied the caustic technique to clusters extracted from a cosmological hydrodynamic simulation of a $\Lambda$ CDM universe, used a different technique (see their Section 4.2). S11 claimed that their algorithm turns out to be more accurate in determining the thresholds since it improves the $\sigma_{\text {caus }} / \sigma_{\text {true }}$ relation (see their Figure 9).

The picture, however, can be more complicated since a few levels of plateaus with different lengths can be seen in data due to substructure. Here we use a similar approach to $\mathrm{S} 11$, but taking into consideration that a few plateaus can be present in the $\sigma_{x}$ versus $x$ diagram. The $\sigma_{p l}$ node region location is determined by the minimum ratio between the standard deviation and average of the $\sigma_{x}$ over a range of at least $N_{\text {node,min }}$ number of (main branch) nodes, i.e.,

$$
\delta_{\min }=\min \left\{\frac{\Delta \sigma_{x_{1}, x_{2}}}{\left\langle\sigma_{x_{1}, x_{2}}\right\rangle}\right\}_{\mathrm{N} \geqslant \mathrm{N}_{\mathrm{nod}, \min }},
$$

where we take $N_{\text {node, min }}=7$ to have a minimal number of galaxies for the standard deviation estimation. The cluster identifying node, i.e., $x_{2}$, is determined by the largest node that is below $\sigma_{p l}\left(1+\# \delta_{\min }\right)$, where \# was taken to be 2 .

\section{B.2.3. Building the Redshift-phase Diagram}

The galaxies hanging from node $x_{2}$ and which constitute the main group of the binary tree determine the velocity dispersion and mean projected distance of the members. These two are then used in the D99 procedure, which locates the caustics using all the galaxies and determines the radial dependence of their amplitude (in units of velocity). Galaxies that are inside the caustics are considered to be cluster members. Of course, some of these galaxies might still be interlopers, but their number is typically a few percent and has little effect on dynamical analyses (Serra \& Diaferio 2013; A. Diaferio 2012, private communication).

In more details, a redshift-space diagram is constructed from all the galaxies after the velocity cut (see Section 3.1). Then we determine the threshold $\kappa$ that defines the caustic location through $f(R, v)=\kappa$. Here $f(R, v)$ is the galaxy density distribution in the redshift-space diagram, smoothed with a multidimensional adaptive kernel (Silverman 1986; Pisani 1993; Pisani 1996). ${ }^{37}$ For the calculation of the optimal smoothing

\footnotetext{
37 Gifford et al. (2013), who used simulations, claim that a standard fixed kernel also recovers the cluster mass estimates with low scatter and bias.
} 
length (see D99, Equation (20)), the two coordinates $R$ and $v$ must have the same units. Therefore, we divide them by their maximum value. In addition, Silverman (1986, p. 77) suggests rescaling these coordinates to avoid extreme differences of spread in the various coordinate directions, so we further divide them by their dispersion. The maximum and dispersion of both $R$ and $v$ are determined from the cluster member candidates. ${ }^{38}$

The parameter $\kappa$ is chosen by minimizing the quantity $S(\kappa,\langle R\rangle)=\left|\left\langle v_{\mathrm{esc}}^{2}\right\rangle_{\kappa,\langle R\rangle}-4\left\langle v^{2}\right\rangle\right|^{2}$, where $\left\langle v_{\mathrm{esc}}^{2}\right\rangle_{\kappa,\langle R\rangle}=$ $\int_{0}^{\langle R\rangle} A^{2}(R) \phi(R) d R / \int_{0}^{\langle R\rangle} \phi(R) d R$ is the mean value of the square of the caustic amplitude $A(R)$ within $\langle R\rangle$ (the mean projected radius of the cluster members), $\phi(R)=\int f(R, v) d v$, and $\left\langle v^{2}\right\rangle^{1 / 2}$ is the $1 \mathrm{D}$ velocity dispersion of the cluster members. The caustics are the value of $v(R)$ at the projected radius $R$ at the point where $f(R, v)=\kappa$.

In general, $f(R, v)$ is not symmetric around the cluster redshift, and since we assume spherical symmetry, we take $A(R)=$ $\min \left\{\left|v_{u}(R)\right|,\left|v_{d}(R)\right|\right\}$, where $v_{u}(R)$ and $v_{d}(R)$ are the caustics above and below the clusters' redshift, respectively. Taking the minimum of the upper and lower caustics is more robust than $A(R)=\left(v_{u}(R)-v_{d}(R)\right) / 2$ against interloper contamination and presence of massive substructure. Finally, since for any realistic system $d \ln A / d \ln R \lesssim 1 / 4$, D99 claimed that to control the contamination by background and foreground galaxies efficiently a further step is needed. We follow D99 and accept only values of $A(R)$ that yield $\frac{d \ln A}{d \ln R} \leqslant \zeta_{\text {caustics, }}$, when $\zeta_{\text {caustics }}=$ $1 / 4$. Otherwise, we impose new values of $A(R)$ that yield $d \ln A / d \ln R=1 / 4$. Note that D99 and S11 took $\zeta_{\text {caustics }}=1$ and $\zeta_{\text {caustics }}=2$, respectively.

In Table 3, we also present the clusters' redshift estimations as suggested in D99. The cluster center in redshift space is the median of the cluster member velocity distribution.

Caustic uncertainty. A larger $\kappa$ indicates a smaller number of galaxies within the caustics and therefore a larger uncertainty. Similarly, a poorly sampled cluster yields a small $\max \{f(R, v)\}$ and therefore, again, a large uncertainty. If the area surrounding the caustics is poorly populated and the area within the caustics is well populated, $\kappa$ is low and $\max \{f(R, v)\}$ is large and the uncertainty is small. Therefore, the caustic uncertainty is taken to be $d A(R) / A(R)=\kappa / \max \{f(R, v)\}$, where the maximum is found along the $v$-axis at each $R$ (D99; A. Diaferio 2012, private communication). ${ }^{39}$

\section{B.2.4. The Value of $F_{\beta}$}

In order to calculate the caustic mass (see Equation (7)), one has to set a value for $F_{\beta}$. This factor, which absorbs in it

\footnotetext{
38 Previously, in the caustic method, one of the major input parameters was the rescaling parameter, $q$, which sets the scaling between the quantities $R$ and $v$ within the smoothing procedure (D99). There is no simple a priori choice for this parameter value. Usually it was chosen to be $q=25$ (e.g., D99; Rines et al. 2003), which was obtained by the ratio of the two coordinates' uncertainties (D99). It was claimed that different values of $q$ in the 10-50 range have little effect on the results (D99; Rines et al. 2002). However, even in this $q$ range, Rines et al. (2002) tested the effect of different $q$ values on the A2199 supercluster estimated mass profile and found that although the mass mean does not depend strongly on $q$, the mass uncertainty does. In addition, Reisenegger et al. (2000) found that $q$ values out of the 10-50 range are more suitable in the case of the Shapley supercluster. In this paper, we rescale $R$ and $v$ in an automatic procedure instead of choosing the scaling by hand.

39 By analyzing a sample of 3000 simulated clusters with masses of $M_{200}>10^{14} h_{0.73}^{-1} M_{\odot}, \mathrm{S} 11$ tested the deviation of this recipe from the $1 \sigma$ confidence level. They found that on average at $r_{200}$ the upper caustic uncertainty is underestimated by about $24 \%$ and the lower caustic uncertainty is overestimated by about a few percent (see left panel in their Figure 16; A. L. Serra 2012-2013, private communication).
}

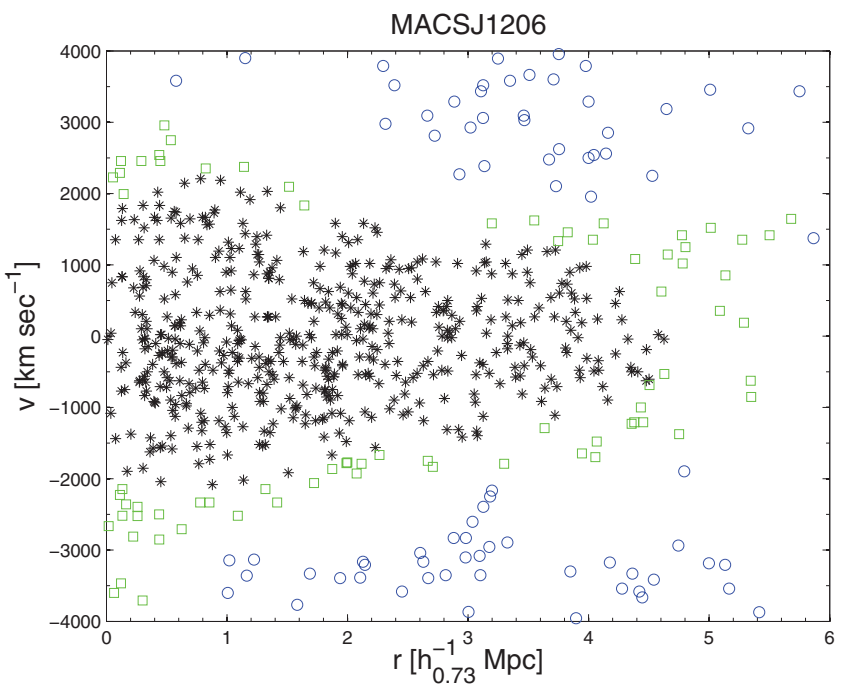

Figure 12. Phase-space diagram of MACSJ1206. Galaxies which are left after cleaning interlopers using the D99 method are marked by black asterisks. Galaxies that are left after cleaning interlopers using the HK96 method but are considered interlopers using the D99 method are marked by green squares. Galaxies that are considered to be interlopers by both the D99 and HK96 methods are marked by blue circles.

(A color version of this figure is available in the online journal.)

the galaxy velocity anisotropy profile $(\beta),{ }^{40}$ varies slowly with the radius and, therefore, is taken to be constant (D99). D99 followed Diaferio \& Geller (1997) and set $F_{\beta}$ to be 0.5 , finding that the resulting caustic method recovers the true cluster mass within a factor of 2 at large radii, $r \sim(0.3-6) r_{200}$, at least with the low concentration parameters of his simulated clusters. D99's hyperbolic fit to the $\sigma_{x}-x$ diagram puts the thresholds $\left(x_{1}\right.$ and $x_{2}$ ) below (in the case of substructure threshold) and above (in the case of the cluster threshold) the plateau in most cases. An increase in $x_{2}$ increases the cluster galaxies' velocity dispersion (for more details, see Appendix B.2). As a result, the caustics are wider. The S11 algorithm places $x_{1}$ and $x_{2}$ on the plateau, which means that the caustics are slightly narrower (A. L. Serra 2012-2013, private communication). To compensate for that, they adopted a higher value for $F_{\beta}, 0.7$, instead of $0.5 .^{41}$ This large difference in the value of $F_{\beta}$ is because the caustics are sensitive to the $\left\langle v^{2}\right\rangle$ value (D99), which depends on the binary tree cutting method. Since our threshold-finding algorithm is closer to the one used in S11 (see Appendix B.2), we adopted $F_{\beta}=0.7$.

In an independent work from S11, Gifford et al. (2013) used simulations and also found a high value for the caustic mass scaling factor, $F_{\beta}=0.65$. They also gave another explanation for the $0.5-0.7$ scatter in the $F_{\beta}$ values (see their Section 4.3).

\section{B.3. Velocity-space Diagram for MACSJ1206}

In this section, we show the velocity-space diagram (see Section 3.2) of MACSJ1206, which has the highest number

\footnotetext{
40 The $\beta$, in this subsection, is not to be confused with the $\beta$ parameter in Equation (16).

41 Lemze et al. (2009), who analyzed A1689 using galaxy dynamics, derived the cluster's $F_{\beta}$ profile (see their Figure 9), using the dynamical information and the mass density profile derived in Lemze et al. (2008). However, they did not attempt to constrain the cluster's $F_{\beta}$ mean value because, at that time, the only value suggested in previous works was 0.5 . Even if the mean $F_{\beta}$ was constrained for this cluster, a large scatter is expected between the $F_{\beta}$ profiles of different clusters (D99; S11). Thus, the mean $F_{\beta}$ of A1689 can be different than that averaged over many clusters.
} 


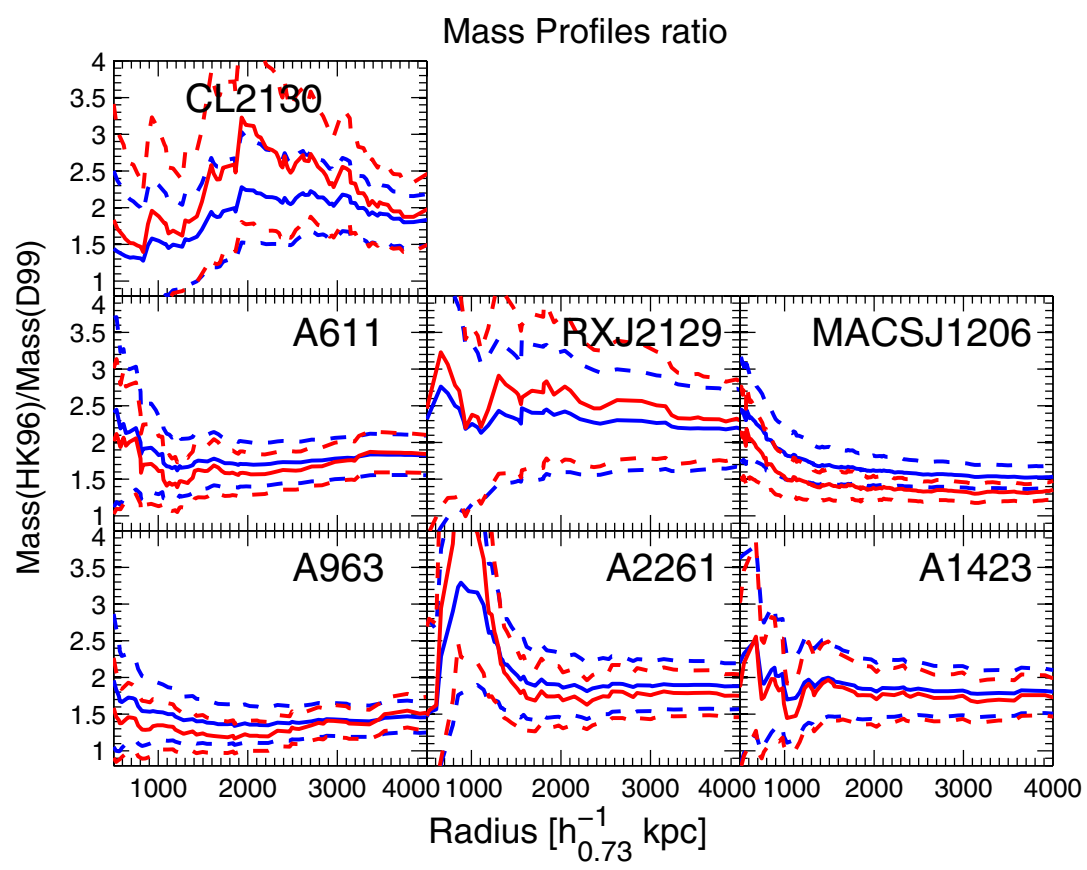

Figure 13. Mass profile ratio inferred by using different interloper removal methods, HK96 and D99. Blue and red curves are for the corrected virial and projected mass profiles, respectively. The upper and lower dashed curves represent the $\pm 1 \sigma$ width of the distribution uncertainty.

(A color version of this figure is available in the online journal.)
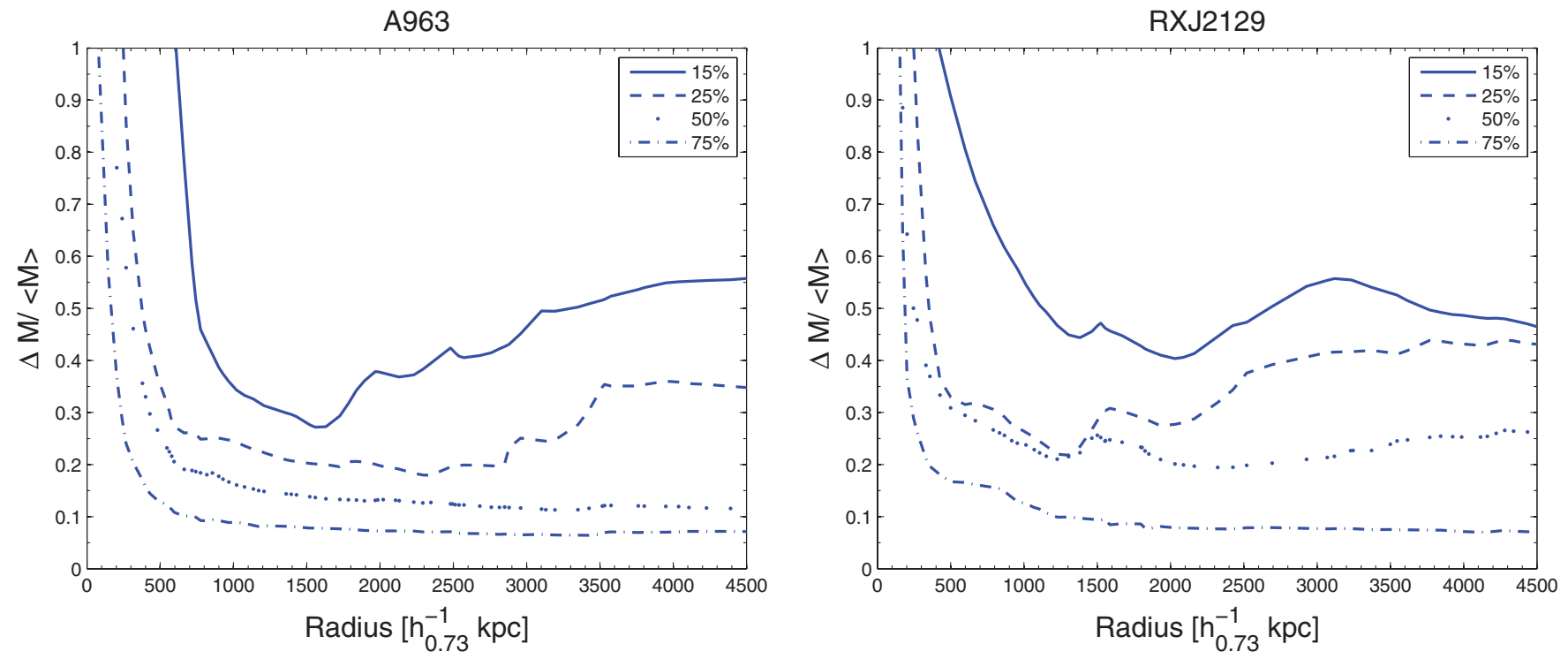

Figure 14. Statistical uncertainty due to the number of galaxies. The statistical uncertainty is estimated by randomly taking a fraction of the galaxy number after the initial velocity cut (see Section 3.1), removing interlopers using the HK96 method, and estimating the corrected virial mass profile. This procedure is repeated 50 times for each fraction, and the statistical uncertainty is estimated by dividing the scatter by the mean of all the repetitions. Left panel: taking different galaxy fractions from A963 galaxies. Solid, dashed, dotted, and dot-dashed curves are for taking 15\%, 35\%, 50\%, and 75\% of the galaxies, respectively. Right panel: the same as the left panel but for RXJ2129.

(A color version of this figure is available in the online journal.)

of bound galaxies in our sample. In the velocity-space diagram, we clean interlopers using the two different methods: HK96 and D99 (see also Biviano et al. (submitted), who used two other techniques to remove interlopers from this cluster). In Figure 12, galaxies which are left after cleaning interlopers using the D99 method are marked by black asterisks. Galaxies which are left after cleaning interlopers using the HK96 method but are considered interlopers using the D99 method are marked by green squares. Galaxies which are considered to be interlopers by both the D99 and HK96 methods are marked by blue circles.

\section{APPENDIX C}

\section{MASS PROFILE BIASES}

In this section, we examine various sources that may affect the mass profile (see also Biviano et al. 2006), irrespective of the mass estimator used. The first one is the dependence of the mass profile on the interloper removal method. In Figure 13, we plotted the ratio between the mass profiles when using the HK96 and D99 interloper removal methods (for more details, 

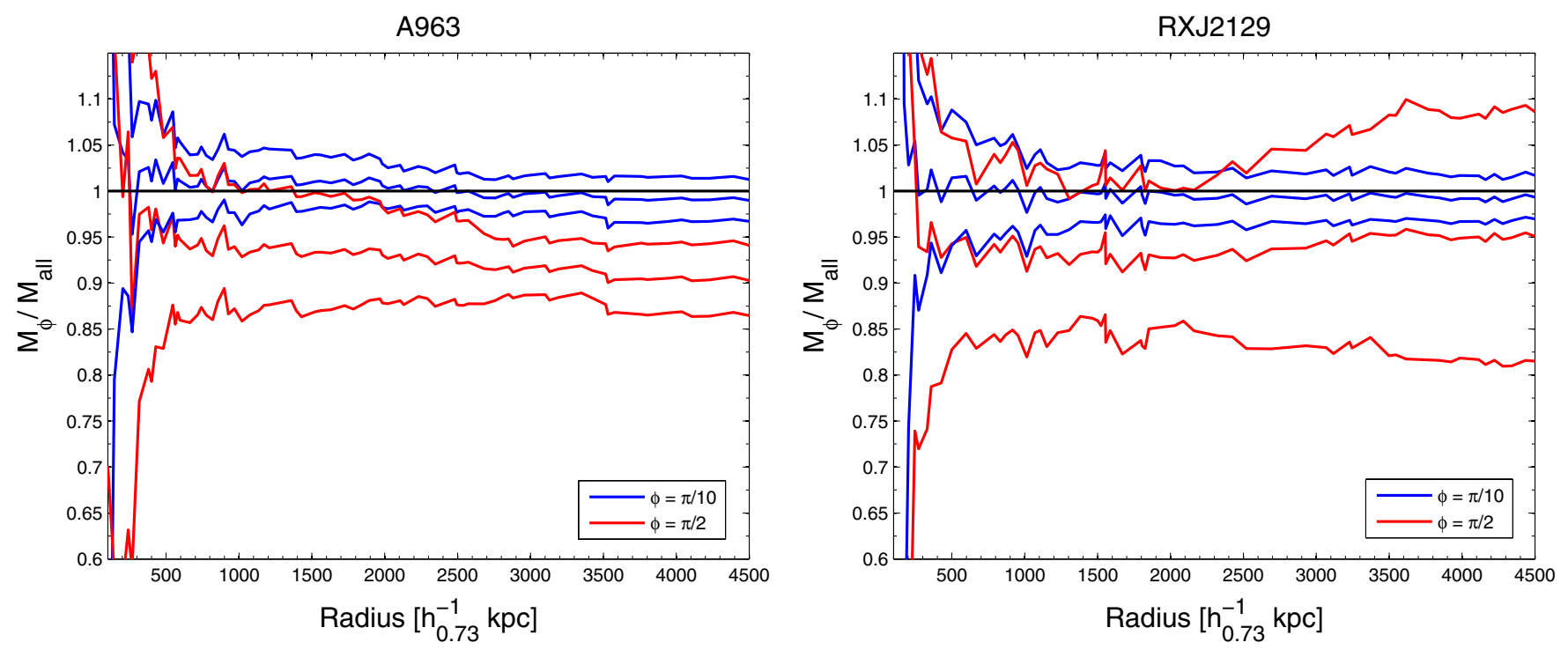

Figure 15. Mass profile bias due to uneven sampling of the plane of the sky. We estimate the virial mass profile after excluding galaxies in a sector with opening angles of $\phi=\pi / 10$ (blue curves) and $\pi / 2$ (red curves) and removing interlopers using the HK96 method. For each $\phi$, the mass profile, $M(\phi)$, is estimated $N_{\theta}=50$ times, where in each time the sector is rotated by an angle $\theta$, so $\theta \times N_{\theta}$ gives $2 \pi$. Left panel is for A963, and right panel is for RXJ2129. In both figures, the middle solid and the upper and lower solid curves represent the mean and $1 \sigma$ scatter, respectively, of all rotations.

(A color version of this figure is available in the online journal.)

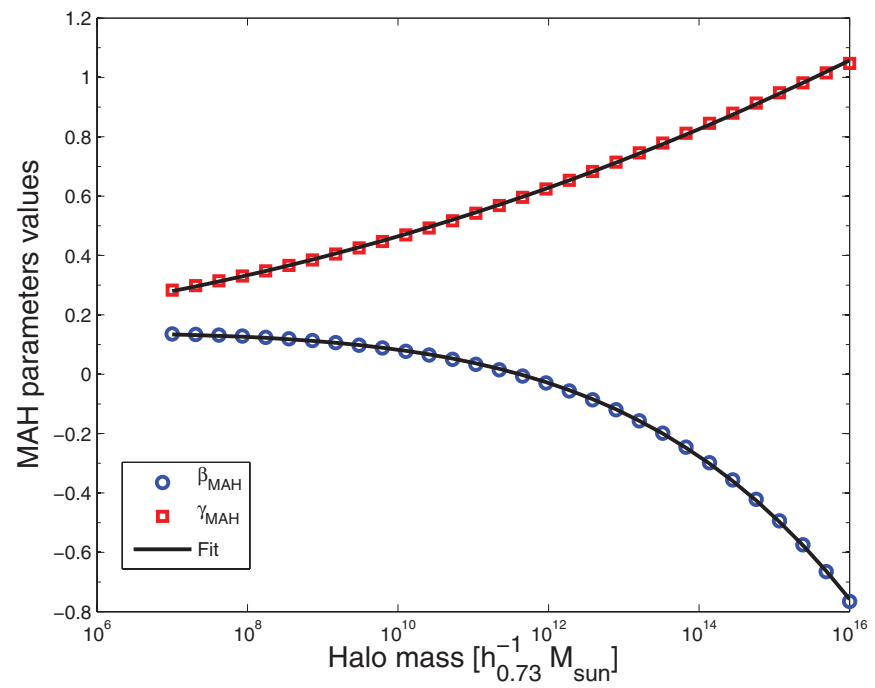

Figure 16. MAH parameter fit. Blue circles and red squares are for $\beta_{\mathrm{MAH}}$ and $\gamma_{\mathrm{MAH}}$, respectively. These are obtained by fitting Equation (13) to the MAH derived using Equation (15). Black solid lines are the fit to $\beta_{\mathrm{MAH}}$ and $\gamma_{\mathrm{MAH}}$ using Equation (D1).

(A color version of this figure is available in the online journal.)

see Section 3.2). We show this ratio for both the corrected virial (blue curve) and projected (red curve) mass profiles.

A second test we make is to estimate the statistical uncertainty due to the number of spectroscopic identified galaxies. We randomly take a fraction of the galaxy number after the initial velocity cut (see Section 3.1). Then we remove interlopers using the HK96 method and estimate the corrected virial mass profile. This procedure is done 50 times, and the statistical uncertainty due to the number of galaxies is estimated as the scatter divided by the mean. In Figure 14, we show the results of this test for A963 and RXJ2129, and for a few different fractions of the spectroscopic identified galaxy number. Solid, dashed, dotted, and dot-dashed curves are for taking 15\% (when these $15 \%$ are 41 and 63 galaxies for A963 and RXJ2129, respectively), 35\%, $50 \%$, and $75 \%$ of the galaxies, respectively.
Lastly, we estimate the uncertainty in the virial mass profile due to plane of the sky incompleteness sampling. We take our spectroscopic data, exclude part of them, cleaning them from interlopers using the HK96 procedure, and estimate the virial mass profile. The excluded part is taken to be a sector centered at the cluster center and with an opening angle $\phi$. Assuming a relaxed and spherical cluster, mass profiles estimated from different sectors should be the same. Clusters, on the other hand, may not be completely relaxed or spherical. However, the level of relaxation can be quantified (as we show in Section 3.5.2), and the ellipticity effect can be averaged out when taking a sample of clusters, each with a different ellipticity. In Figure 15, we plot the virial mass profiles after excluding galaxies in sectors with opening angles of $\phi=\pi / 10$ (blue curves) and $\pi / 2$ (red curves). If we exclude only an annulus within the sector, the bias is obviously smaller than excluding the whole sector. If we exclude an inner annulus, the bias is larger than excluding an outer annulus (not shown) since the galaxies' density is higher closer to the cluster center. For each $\phi$, the mass profile, $M(\phi)$, is estimated $N_{\theta}=50$ times, where in each time the sector is rotated by an angle $\theta$, so $\theta \times N_{\theta}$ gives $2 \pi$. The mass uncertainty from each sector is taken to be the standard deviation over all $\theta$.

\section{APPENDIX D}

$$
\text { ESTIMATING } \beta_{\mathrm{MAH}}\left(M_{0}\right) \text { AND } \gamma_{\mathrm{MAH}}\left(M_{0}\right)
$$

Here we explicitly express $\beta_{\mathrm{MAH}}\left(M_{0}\right)$ and $\gamma_{\mathrm{MAH}}\left(M_{0}\right)$ as functions of $M_{0}$. This allows us together with Equation (13) to calculate the averaged halos' MAH for any $M_{0}$. In order to do so, we first derive $\beta_{\mathrm{MAH}}$ and $\gamma_{\mathrm{MAH}}$ for different $M_{0}$ values. For each $M_{0}$, we fit the expression given in Equation (13) to the mass histories inferred from the mean mass growth rates of halos, i.e., Equation (15). Then, we fit the following phenomenological functions to the $\beta_{\mathrm{MAH}}$ and $\gamma_{\mathrm{MAH}}$ obtained for the different $M_{0}$ values:

$$
\begin{aligned}
& \beta_{\mathrm{MAH}}\left(M_{0}\right)=C_{1}\left(1+\left(\log \left(M_{0}\right) / C_{2}\right)^{C_{3}}\right)^{-1}+C_{4} \\
& \gamma_{\mathrm{MAH}}\left(M_{0}\right)=C_{1}\left(1+\left(\log \left(M_{0}\right) / C_{2}\right)^{C_{3}}\right)+C_{4},
\end{aligned}
$$


where $C_{1}, C_{2}, C_{3}$, and $C_{4}$ are the fitted expressions' free parameters. The best-fit values are $C_{1}=(28.2684,6.3862)$, $C_{2}=(28.6379,39.5429), C_{3}=(5.8674,2.1171)$, and $C_{4}=$ $(-28.1271,-6.2693)$, when the left and right values are for $\beta_{\mathrm{MAH}}$ and $\gamma_{\mathrm{MAH}}$, respectively. In Figure 16 , we plot $\beta_{\mathrm{MAH}}$ and $\gamma_{\text {MAH }}$ as blue circles and red squares, respectively. On top, we plot the fitted expression as a black solid line.

\section{REFERENCES}

Abazajian, K. N., Adelman-McCarthy, J. K., Agüeros, M. A., et al. 2009, ApJS, 182,543

Adami, C., Biviano, A., Durret, F., \& Mazure, A. 2005, A\&A, 443, 17

Angulo, R. E., Springel, V., White, S. D. M., et al. 2012, MNRAS, 425, 2722

Angulo, R. E., \& White, S. D. M. 2010, MNRAS, 405, 143

Arnaud, M., Pratt, G. W., Piffaretti, R., et al. 2010, A\&A, 517, A92

Bahcall, J. N., \& Tremaine, S. 1981, ApJ, 244, 805

Bennett, C. L., Larson, D., Weiland, J. L., et al. 2013, ApJS, 208, 20

Berrier, J. C., Stewart, K. R., Bullock, J. S., et al. 2009, ApJ, 690, 1292

Bertschinger, E., \& Gelb, J. M. 1991, ComPh, 5, 164

Binney, J., \& Tremaine, S. (ed.) 2008, Galactic Dynamics (2nd ed.; Princeton, NJ: Princeton Univ. Press)

Bird, C. M. 1994, AJ, 107, 1637

Biviano, A., Katgert, P., Thomas, T., \& Adami, C. 2002, A\&A, 387, 8

Biviano, A., Murante, G., Borgani, S., et al. 2006, A\&A, 456, 23

Boylan-Kolchin, M., Springel, V., White, S. D. M., Jenkins, A., \& Lemson, G. 2009, MNRAS, 398, 1150

Bryan, G. L., \& Norman, M. L. 1998, ApJ, 495, 80

Busha, M. T., Adams, F. C., Wechsler, R. H., \& Evrard, A. E. 2003, ApJ, 596,713

Carlberg, R. G., Yee, H. K. C., \& Ellingson, E. 1997, ApJ, 478, 462

Carlberg, R. G., Yee, H. K. C., Ellingson, E., et al. 1996, ApJ, 462, 32

Cavagnolo, K. W., Donahue, M., Voit, G. M., \& Sun, M. 2009, ApJS, 182, 12

Chandrasekhar, S. 1961, International Series of Monographs on Physics (Oxford: Clarendon)

Coe, D., Umetsu, K., Zitrin, A., et al. 2012, ApJ, 757, 22

Dai, X., Bregman, J. N., Kochanek, C. S., \& Rasia, E. 2010, ApJ, 719, 119

Davis, A. J., D'Aloisio, A., \& Natarajan, P. 2011, MNRAS, 416, 242

Davis, M., Efstathiou, G., Frenk, C. S., \& White, S. D. M. 1985, ApJ, 292, 371

den Hartog, R., \& Katgert, P. 1996, MNRAS, 279, 349

Diaferio, A. 1999, MNRAS, 309, 610

Diaferio, A., \& Geller, M. J. 1997, ApJ, 481, 633

Dressler, A., Bigelow, B., Hare, T., et al. 2011, PASP, 123, 288

Dressler, A., \& Shectman, S. A. 1988, AJ, 95, 985

Dünner, R., Araya, P. A., Meza, A., \& Reisenegger, A. 2006, MNRAS, 366, 803

Ebeling, H., Ma, C. J., Kneib, J.-P., et al. 2009, MNRAS, 395, 1213

Efron, B. 1981, Biometrika, 68, 589

Fabricant, D., Fata, R., Roll, J., et al. 2005, PASP, 117, 1411

Fakhouri, O., \& Ma, C.-P. 2008, MNRAS, 386, 577

Fakhouri, O., Ma, C.-P., \& Boylan-Kolchin, M. 2010, MNRAS, 406, 2267

Gelb, J. M., \& Bertschinger, E. 1994, ApJ, 436, 467

Geller, M. J., Diaferio, A., Rines, K. J., \& Serra, A. L. 2013, ApJ, 764, 58

Genel, S., Bouché, N., Naab, T., Sternberg, A., \& Genzel, R. 2010, ApJ, 719,229

Gifford, D., \& Miller, C. J. 2013, ApJL, 768, L32

Gifford, D., Miller, C. J., \& Kern, N. 2013, ApJ, 773, 116

Giodini, S., Pierini, D., Finoguenov, A., et al. 2009, ApJ, 703, 982

Girardi, M., Giuricin, G., Mardirossian, F., Mezzetti, M., \& Boschin, W. 1998, ApJ, 505, 74

Gonzalez, A. H., Zaritsky, D., \& Zabludoff, A. I. 2007, ApJ, 666, 147

Harrison, E. R., \& Noonan, T. W. 1979, ApJ, 232, 18

Heisler, J., Tremaine, S., \& Bahcall, J. N. 1985, ApJ, 298, 8

Hinshaw, G., Larson, D., Komatsu, E., et al. 2013, ApJS, 208, 19
Horner, D. J. 2001, PhD thesis, Univ. Maryland, College Park

Huchra, J. P., \& Geller, M. J. 1982, ApJ, 257, 423

Jones, D. H., Read, M. A., Saunders, W., et al. 2009, MNRAS, 399, 683

Jones, D. H., Saunders, W., Colless, M., et al. 2004, MNRAS, 355, 747

Kaiser, N. 1987, MNRAS, 227, 1

Kirkpatrick, S., Gelatt, C. D., \& Vecchi, M. P. 1983, Sci, 220, 671

Knebe, A., \& Muller, V. 2000, A\&A, 354, 761

Koester, B. P., McKay, T. A., Annis, J., et al. 2007, ApJ, 660, 239

Komatsu, E., Smith, K. M., Dunkley, J., et al. 2011, ApJS, 192, 18

Kravtsov, A. V., Gnedin, O. Y., \& Klypin, A. A. 2004, ApJ, 609, 482

Lacey, C., \& Cole, S. 1994, MNRAS, 271, 676

Lamareille, F., Contini, T., Le Borgne, J.-F., et al. 2006, A\&A, 448, 893

Le Fèvre, O., Saisse, M., Mancini, D., et al. 2003, Proc. SPIE, 4841, 1670

Lemze, D., Barkana, R., Broadhurst, T. J., \& Rephaeli, Y. 2008, MNRAS, 386, 1092

Lemze, D., Broadhurst, T., Rephaeli, Y., Barkana, R., \& Umetsu, K. 2009, ApJ, 701, 1336

Lemze, D., Wagner, R., Rephaeli, Y., et al. 2012, ApJ, 752, 141

Lin, Y.-T., Mohr, J. J., \& Stanford, S. A. 2003, ApJ, 591, 749

Lokas, E. L., \& Hoffman, Y. 2001, arXiv:astro-ph/0108283

Macciò, A. V., Dutton, A. A., van den Bosch, F. C., et al. 2007, MNRAS, 378 , 55

McBride, J., Fakhouri, O., \& Ma, C.-P. 2009, MNRAS, 398, 1858

Nagamine, K., \& Loeb, A. 2003, NewA, 8, 439

Navarro, J. F., Frenk, C. S., \& White, S. D. M. 1997, ApJ, 490, 493

Neto, A. F., Gao, L., Bett, P., et al. 2007, MNRAS, 381, 1450

Newman, A. B., Treu, T., Ellis, R. S., \& Sand, D. J. 2013a, ApJ, 765, 25

Newman, A. B., Treu, T., Ellis, R. S., et al. 2013b, ApJ, 765, 24

Okabe, N., Takada, M., Umetsu, K., Futamase, T., \& Smith, G. P. 2010, PASJ, 62,811

Pinkney, J., Roettiger, K., Burns, J. O., \& Bird, C. M. 1996, ApJS, 104, 1

Pisani, A. 1993, MNRAS, 265, 706

Pisani, A. 1996, MNRAS, 278, 697

Postman, M., Coe, D., Benítez, N., et al. 2012, ApJS, 199, 25

Quenouille, M. H. 1949, J. R. Stat. Soc. B, 11, 68

Regos, E., \& Geller, M. J. 1989, AJ, 98, 755

Reisenegger, A., Quintana, H., Carrasco, E. R., \& Maze, J. 2000, AJ, 120, 523

Rines, K., \& Diaferio, A. 2006, AJ, 132, 1275

Rines, K., Geller, M. J., Diaferio, A., \& Kurtz, M. J. 2013, ApJ, 767, 15

Rines, K., Geller, M. J., Diaferio, A., et al. 2002, AJ, 124, 1266

Rines, K., Geller, M. J., Kurtz, M. J., \& Diaferio, A. 2003, AJ, 126, 2152

Rodríguez-Gonzálvez, T. A. C. C., Shimwell, T. W., Davies, M. L., et al. 2012, MNRAS, 425, 162

Saro, A., Bazin, G., Mohr, J., \& Dolag, K. 2013, ApJ, 772, 47

Serna, A., \& Gerbal, D. 1996, A\&A, 309, 65

Serra, A. L., \& Diaferio, A. 2013, ApJ, 768, 116

Serra, A. L., Diaferio, A., Murante, G., \& Borgani, S. 2011, MNRAS, 412, 800

Shaw, L. D., Weller, J., Ostriker, J. P., \& Bode, P. 2006, ApJ, 646, 815

Silverman, B. W. 1986, Monographs on Statistics and Applied Probability (London: Chapman and Hall)

Spergel, D. N., Verde, L., Peiris, H. V., et al. 2003, ApJS, 148, 175

Springel, V., White, S. D. M., Jenkins, A., et al. 2005, Natur, 435, 629

Stoughton, C., Lupton, R. H., Bernardi, M., et al. 2002, AJ, 123, 485

Takizawa, M., Nagino, R., \& Matsushita, K. 2010, PASJ, 62, 951

Tasitsiomi, A., Kravtsov, A. V., Gottlöber, S., \& Klypin, A. A. 2004, ApJ, 607, 125

The, L. S., \& White, S. D. M. 1986, AJ, 92, 1248

Thomas, P. A., Muanwong, O., Pearce, F. R., et al. 2001, MNRAS, 324, 450

Tonry, J., \& Davis, M. 1979, AJ, 84, 1511

Tukey, J. W. 1958, Ann. Math. Stat., 29, 614

Umetsu, K., Medezinski, E., Nonino, M., et al. 2012, ApJ, 755, 56

Wojtak, R., \& Łokas, E. L. 2010, MNRAS, 408, 2442

Wu, H.-Y., Hahn, O., Wechsler, R. H., Mao, Y.-Y., \& Behroozi, P. S. 2013, ApJ, 763,70 